\title{
Supporting Informtaion
}

Computational Insights into the Conformational Accessibility and Binding Strength of SARS-CoV-2 Spike Protein to Human Angiotensin-Converting Enzyme 2

Cheng Penga,b,\#, Zhengdan Zhu ${ }^{\mathrm{a}, \mathrm{b}, \# \text {, Yulong Shia }}{ }^{\mathrm{a}, \mathrm{b}}$, Xiaoyu Wang ${ }^{\mathrm{a}, \mathrm{c}}$, Kaijie Mu ${ }^{\mathrm{a}, \mathrm{d}}$, Yanqing Yanga,b, Xinben Zhang ${ }^{\mathrm{a}}$, Zhijian Xu $\mathrm{Xu}^{\mathrm{a}, \mathrm{b}, *}$, Weiliang Zhu ${ }^{\mathrm{a}, \mathrm{b},{ }^{*}}$

${ }^{a}$ CAS Key Laboratory of Receptor Research; Drug Discovery and Design Center, Shanghai Institute of Materia Medica, Chinese Academy of Sciences, Shanghai, 201203, China

bSchool of Pharmacy, University of Chinese Academy of Sciences, No.19A Yuquan Road, Beijing, 100049, PR China

${ }^{\mathrm{c} C o l l e g e}$ of Mathematics and Physics, Shanghai University of Electric Power, Shanghai 200090, China.

${ }^{\mathrm{d} N a n o}$ Science and Technology Institute, University of Science and Technology of China, Suzhou, Jiangsu, 215123, China.

Supplementary Methods...........S2

Figures S1 to S14_...............S5

Tables S1 to S8..................S16

References.....................S30 


\section{Supplementary Methods}

Preparation of Complexes. In this study, we have investigated the binding affinities of RBD-ACE2 complex and ACE2-bound S trimer for SARS-CoV-2 and SARS-CoV. For SARS-CoV simulations, the initial structures for RBD-ACE2 complex (PDB ID: $2 \mathrm{AJF}^{1}$ ) and ACE2-bound $\mathrm{S}$ trimers (PDB ID: $6 \mathrm{ACG}^{2}, 6 \mathrm{ACK}^{2}, 6 \mathrm{ACJ}^{2}, 6 \mathrm{CS}^{3}$ ) were downloaded from Protein Data Bank. For SARS-CoV-2 simulations, RBD-ACE2 complex structure was available in PDB and used as initial structure (PDB ID: 6M0J ${ }^{4}$ ). However, the structure of ACE2-bound S trimer is not available yet, structures of the four above ACE2-bound CoV-S were thus served as templates to build the threedimensional (3D) models of ACE2-bound CoV-2-S trimer using SWISS-MODEL server $^{5}$. The structural quality of four homology structures was evaluated by Ramachandran plot (Fig. S14), where less than 2\% residues lie in the outlier regions. In addition, a CoV-2-S/ACE2 superimposed model was constructed by superimposing crystal RBD-ACE2 structure (PDB ID: 6M0J) with a CoV-2-S trimer structure (PDB ID: 6VSB). The four homology structures have small RMSD $(\sim 0.68 \AA)$ to the superimposed model for the two ACE2-unbound chains (RBD down state). Large RMSDs are found for the ACE2-bound chain between the four homology structures and the superimposed model $(5.81,3.50,4.77$, and $6.18 \AA$ for $6 \mathrm{ACG}, 6 \mathrm{ACJ}, 6 \mathrm{CS} 2$ and $6 \mathrm{ACK}$, respectively), which result from their different RBD up states.

Each system was solvated in a cubic box of TIP3P water extended by $12 \AA$ from the solute, with a rational number of counter ions of $\mathrm{Na}^{+}$or $\mathrm{Cl}^{-}$to neutralize the system. Amber ff03 force field ${ }^{6}$ was used to parameterize the protein. To remove bad contacts formed during the system preparation, 10,000 steps of minimization including 5,000 steps of steepest descent minimization and 5,000 steps of conjugate gradient minimization were performed. Then, each system was heated to $300 \mathrm{~K}$ within $0.2 \mathrm{~ns}$ followed by $0.1 \mathrm{~ns}$ of equilibration in NPT ensemble. The minimization, heating, and equilibrium simulations were performed with constraints $\left(10 \mathrm{kcal} / \mathrm{mol} / \AA^{2}\right)$ on heavy atoms with sander program in Amber16.

MD and vsREMD simulation. To evaluate the dynamic interactions of ACE2-RBD, $100 \mathrm{~ns}$ MD simulations were performed for ACE2-RBD complexes at $300 \mathrm{~K}$ with pmemd.cuda in Amber 16. Langevin dynamics was used to control temperature, and the SHAKE algorithm ${ }^{7}$ was applied to fix bonds involving hydrogen atoms. A cutoff distance of $12 \AA$ was applied for van der Waals interactions. The particle mesh Ewald method was applied to address long-range electrostatic interactions ${ }^{8}$. 
To simulate the conformational distribution of the two spike proteins, vsREMD method was used. In the vsREMD, briefly, a set of parallel replicas are simulated in explicit solvent environment at different temperatures, but the solvent-solvent interactions are omitted in exchange attempts compensated by velocity rescaling to reduce the number of replicas required ${ }^{9}$. In this study, 48 replicas were used from 300 to $450 \mathrm{~K}(300.0,302.6,305.2,307.9,310.5,313.2,315.9,318.7,321.4,324.2,327.0$, $329.8,332.7,335.6,338.5,341.4,344.4,347.4,350.4,353.4,356.5,359.6,362.7,365.8$, 369.0, 372.2, 375.4, 378.7, 381.9, 385.3, 388.6, 392.0, 395.4, 398.8, 402.2, 405.7, 409.3, $412.8,416.4,420.0,423.6,427.3,431.0,434.7,438.5,442.3,446.1,450.0)$. The initial structures of 48 replicas for CoV-2-S and CoV-S were evenly extracted from their conformational pathway. Exchanges were attempted every 1000 steps. For each replica, the overall simulation time lasted for $100 \mathrm{~ns}$.

Binding free energy calculation. To evaluate the binding affinity between the CoV-2$\mathrm{S}$ or CoV-S and ACE2, the MM/GBSA method was used to calculate the binding free energy $(\Delta G)$ with equation $(\mathrm{S} 1)$ :

$$
\Delta G=\Delta H-T \Delta S=\Delta E_{e l e}+\Delta E_{v d W}+\Delta G_{g b}+\Delta G_{n p}-T \Delta S
$$

where $\Delta E_{\text {ele }}$ and $\Delta E_{v d W}$ are the electrostatic and van der Waals energy terms, and $\Delta G_{\mathrm{gb}}$ and $\Delta G_{\mathrm{np}}$ are the polar and nonpolar solvation free energies, respectively. The Nmode module in Amber16 was used to calculate the conformational entropy $(T \Delta S)$. In this study, OBC solvation model (igb $=5$, mbondi 2$)^{10}$ was applied. The dielectric constant of solvent was set to 80.0. Since the dielectric constant of solute $\left(\varepsilon_{\text {in }}\right)$ has a significant effect on MM/GBSA calculations, simulations with three different $\varepsilon_{\text {in }}\left(\varepsilon_{\text {in }}=1,2,4\right)$ were performed. Based on the calculated $\Delta G$ (Table S8), CoV-2-S was found to always have stronger binding affinity to ACE2 than $\mathrm{CoV}-\mathrm{S}$ at different $\varepsilon_{\text {in }}$ values regardless of RBDangles, although the detail values have obvious difference. Taking into account of the reports by Hou et al, ${ }^{11} \varepsilon_{\text {in }}$ was set to 1.0 in subsequent MM/GBSA calculations. The salt concentration was used to $0.1 M$ mimick a physiological environment. The $\Delta G_{n p}$ was calculated by equation (S2):

$$
\Delta G_{n p}=\gamma \mathrm{SASA}+\beta
$$

where the solvent-accessible surface area (SASA) was calculated with $\gamma=0.0072$ $\mathrm{kcal} / \mathrm{mol} / \AA 2$ and $\beta=0.0 \mathrm{kcal} / \mathrm{mol}$, respectively. To find out crucial residues in the binding of S protein with ACE2, the binding affinity was further decomposed into energy contribution of each residue in Amber16 (idecomp =1).

Conformational change pathway prediction. The "down" to "up" conformational 
change pathway of the CoV-2-S was generated by the NUMD method, of which the details have been described in our previous study ${ }^{12}$. Briefly, many iterations of normal modes analysis (NMA) were run to predict the conformational changes from the initial structure to the final target structure gradually. For example, the intermediate structure $R^{(k)}$ in iteration $k$ was generated by the following equation based on the intermediate structure $R^{(k-1)}$ in iteration $(k-1)$ :

$$
R^{(k)}=R^{(k-1)}+v^{(k)}=R^{(k-1)}+S^{(k)} \sum_{i}^{m^{(k)}}\left(d^{(k-1)} \cdot u_{i}^{(k)}\right) u_{i}^{(k)}
$$

where $v^{(k)}$ is the displacement combined with $m^{(k)}$ low-frequency eigenmodes that are calculated by NMA. For the $i_{t h}$ eigenmode, its displacement is proportional to the projection $d^{(k-1)} u_{i}^{(k)}$, where $d^{(k-1)}$ is the instantaneous distance vector on eigenvector $u_{i}^{(k)}$, and scaled by the step size $S^{(k)}$. In this study, the step size was set to be 10.0 , consistent with our previous study ${ }^{21}$. The starting and final structures were obtained from homology modeling based on the 3D structures of $5 \mathrm{X} 58^{13}$ ("down" conformation with RBD-angle of $31.6^{\circ}$ ) and $5 X 5 B^{13}$ ("up" conformation with RBD-angle of $84.8^{\circ}$ ), respectively, chosen from Table S1 with the highest resolution. To evaluate potential ligand-binding sites for molecular docking in CoV-2-S, we applied the D3Pockets to analyzed the dynamic properties of potential binding sites (https://www.d3pharma.com/D3Pocket/index.php).

Adaptive steered molecular dynamics (ASMD) simulation. In $\mathrm{ASMD}^{14}$, the defined reaction path is divided into $N$ incremental segments. In each segment, the potential of mean force (PMF) along the reaction path is calculated by averaged work distribution $G$ according to Jarzynski's equality,

$\Delta G_{\tau_{t} \leftarrow \tau_{0}}=-\frac{1}{\beta} \ln \left\langle e^{-\beta W_{\tau_{t} \leftarrow \tau_{0}}}\right\rangle_{0}$

where $\beta=1 /\left(k_{B} T\right), t$ is the $t_{t h}$ segment, the work $W \tau_{\mathrm{t}} \leftarrow \tau_{0}$ is done to move the system to $t$ from its initial state. In each segment, the trajectory that has the closest $W \tau_{\mathrm{t}} \leftarrow \tau_{0}$ to PMF of this segment is selected as the initial configuration of next segment. Finally, the iterated average free energy at $\tau_{N}$ could be obtained by equation (S5).

$\Delta G_{\tau_{N} \leftarrow \tau_{0}}=-\frac{1}{\beta} \sum_{i=1}^{N} \ln \left\langle e^{-\beta W_{\tau_{i} \leftarrow \tau_{i-1}}}\right\rangle_{i-1}$

In this study, each segment in ASMD simulations consisted of 20 independent nonequilibrium MD simulations. The steering reaction path was defined by RBD-angles, ranging from $30^{\circ}$ to $85^{\circ} .40 \mathrm{rad} / \mathrm{ns}$ pulling velocity was applied to steer the RBDangle from down to up. The spring constant was set as $50 \mathrm{kcal} / \mathrm{mol} / \mathrm{rad}^{2}$. The identical parameters were used for simulations of glycosylated CoV-2-S and glycosylated $\mathrm{CoV}-\mathrm{S}$ so as to render comparable results. 


\section{Supplementary Figures}

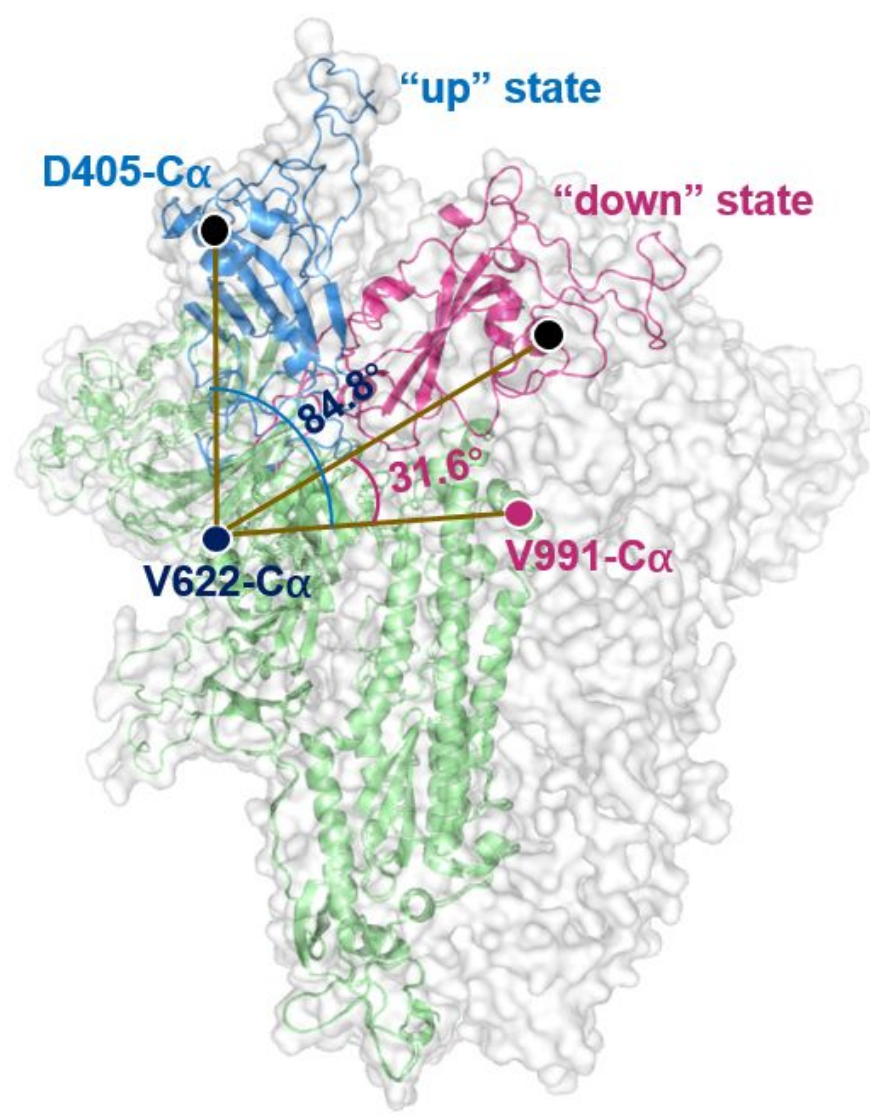

Figure S1. Structures of SARS-CoV-2 spike (CoV-2-S). The schematic diagram of RBD-angle, where the RBD-angle is defined as the angle composed of the three $\mathrm{C} \alpha$ atoms of the residues D405-V622-V991, which corresponds to the angle of D392T608-V973 in CoV-S. 

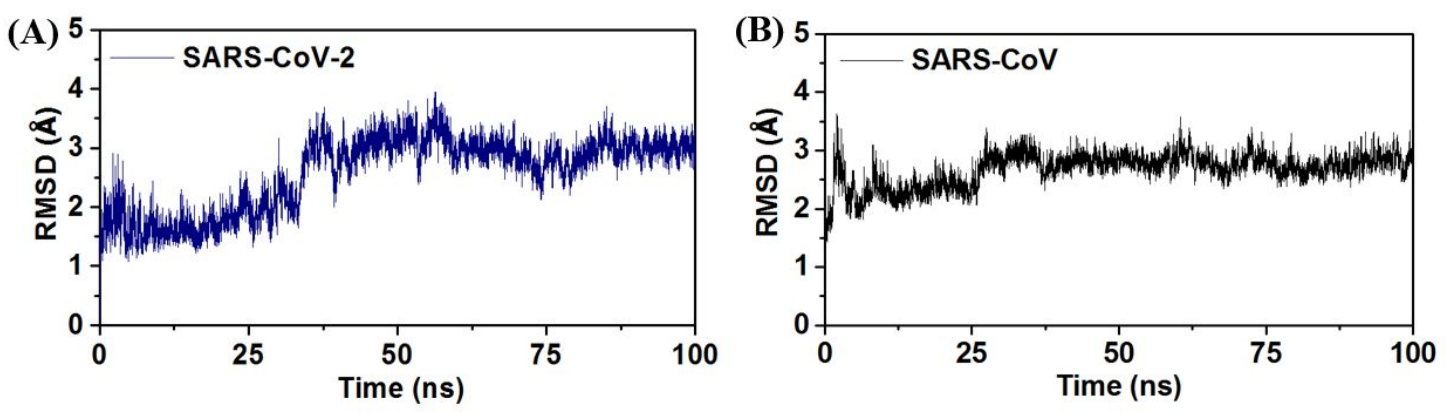

Figure S2. The backbone RMSD of RBD-ACE2 complexes for SARS-CoV-2 (A) and SARS-CoV (B) during $100 \mathrm{~ns}$ MD simulations. 


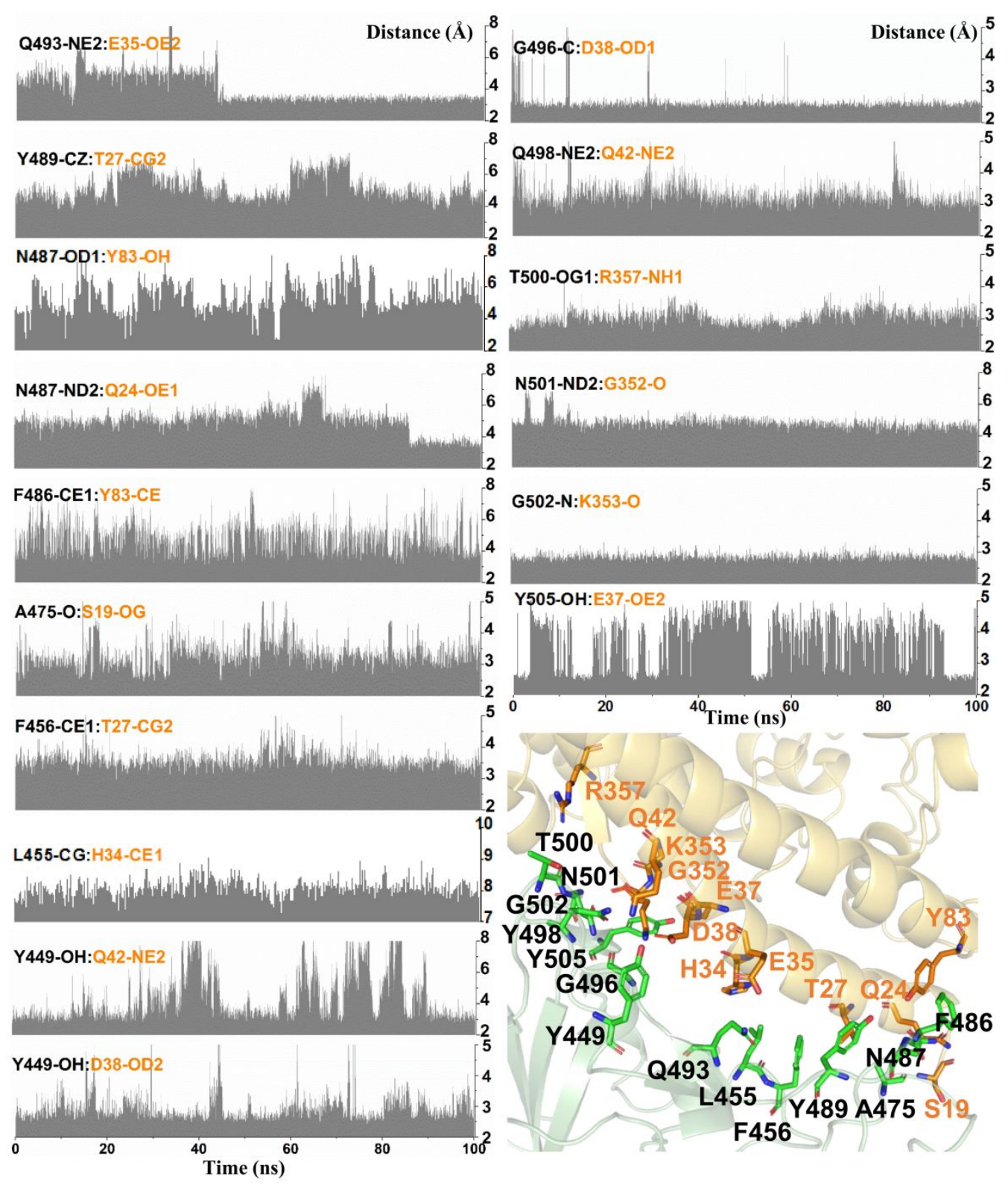

Figure S3: The distance analyses of CoV-2-S and ACE2 interactions along the $100 \mathrm{~ns}$ MD trajectory. Key interactional residues of the CoV-2-S (green) and ACE2 (orange) were shown in sticks. 


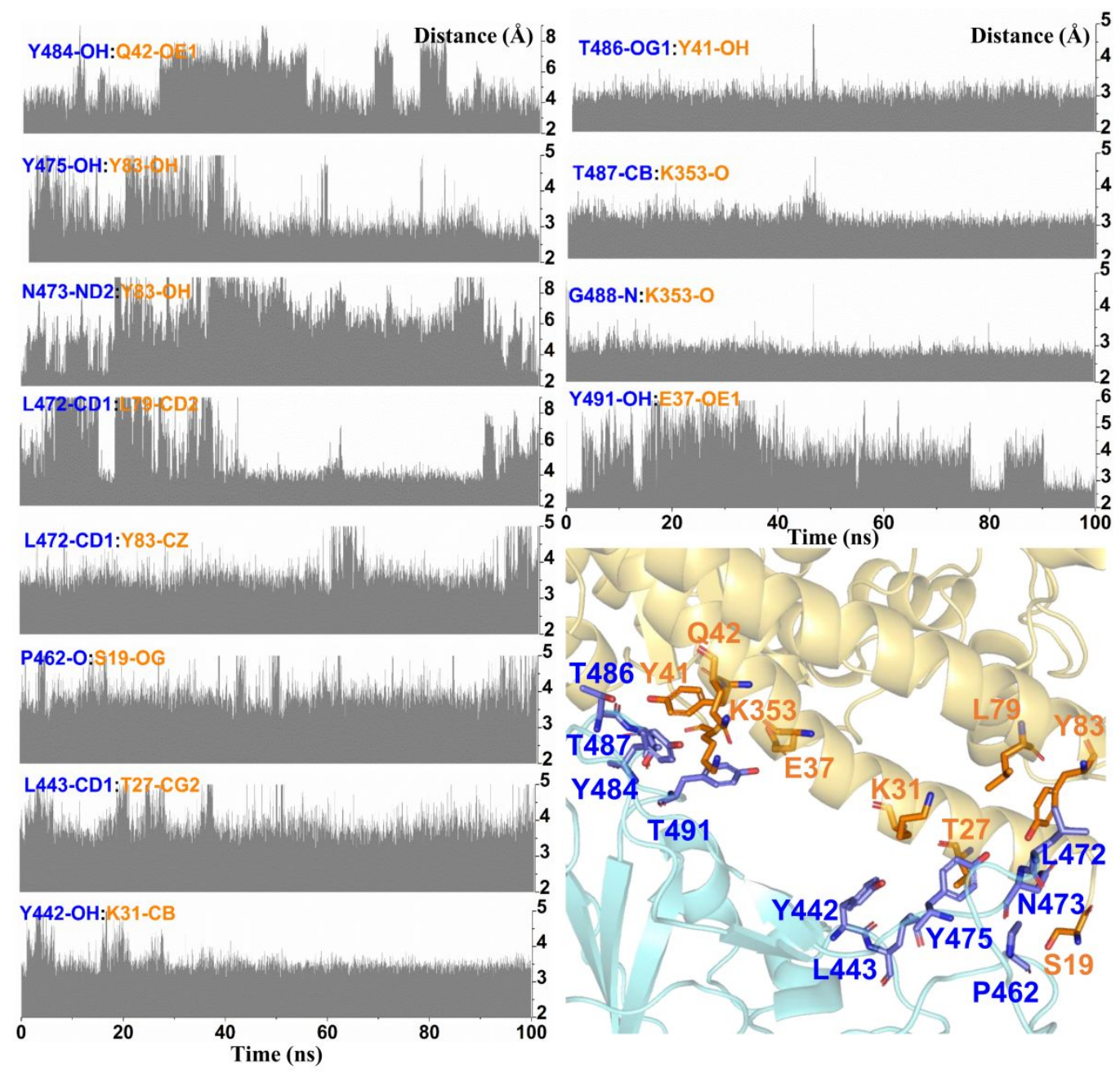

Figure S4: The distance analyses of CoV-S and ACE2 interactions along the $100 \mathrm{~ns}$ MD trajectory. Key interactional residues of the CoV-S (blue) and ACE2 (orange) were shown in sticks. 

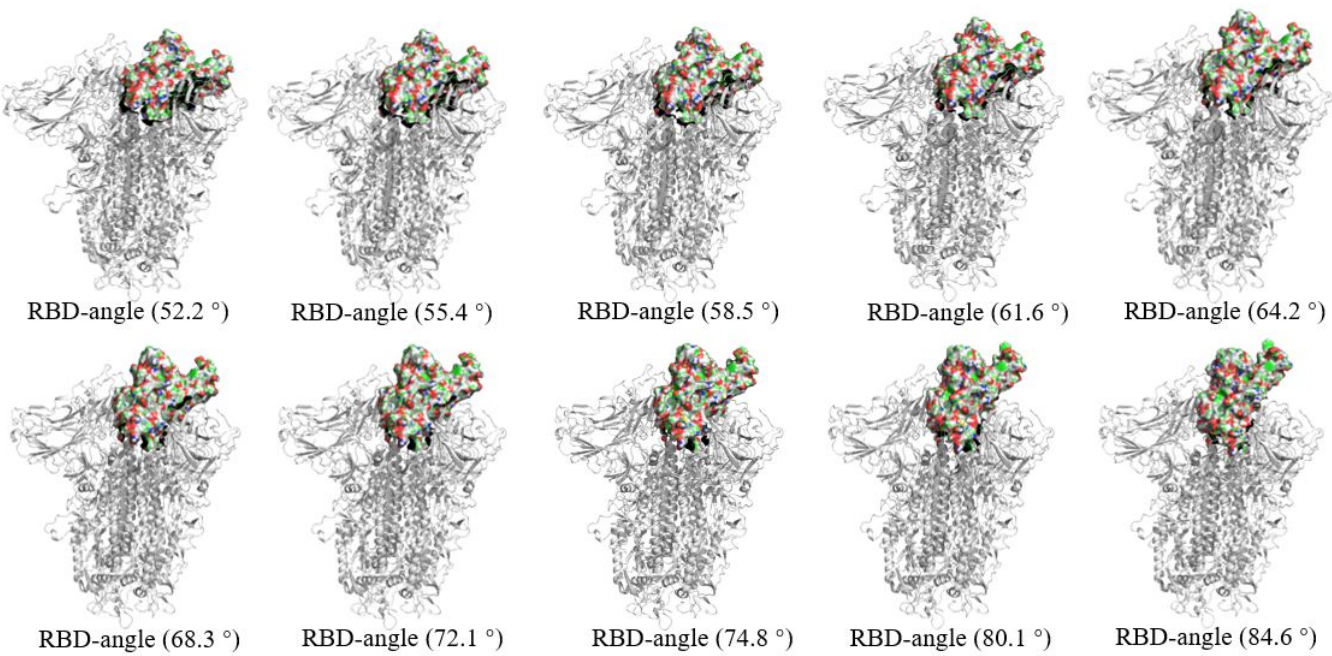

Figure S5. 10 selected conformations of CoV-2-S with different RDB-angles along the "down" to "up" conformational change pathway.

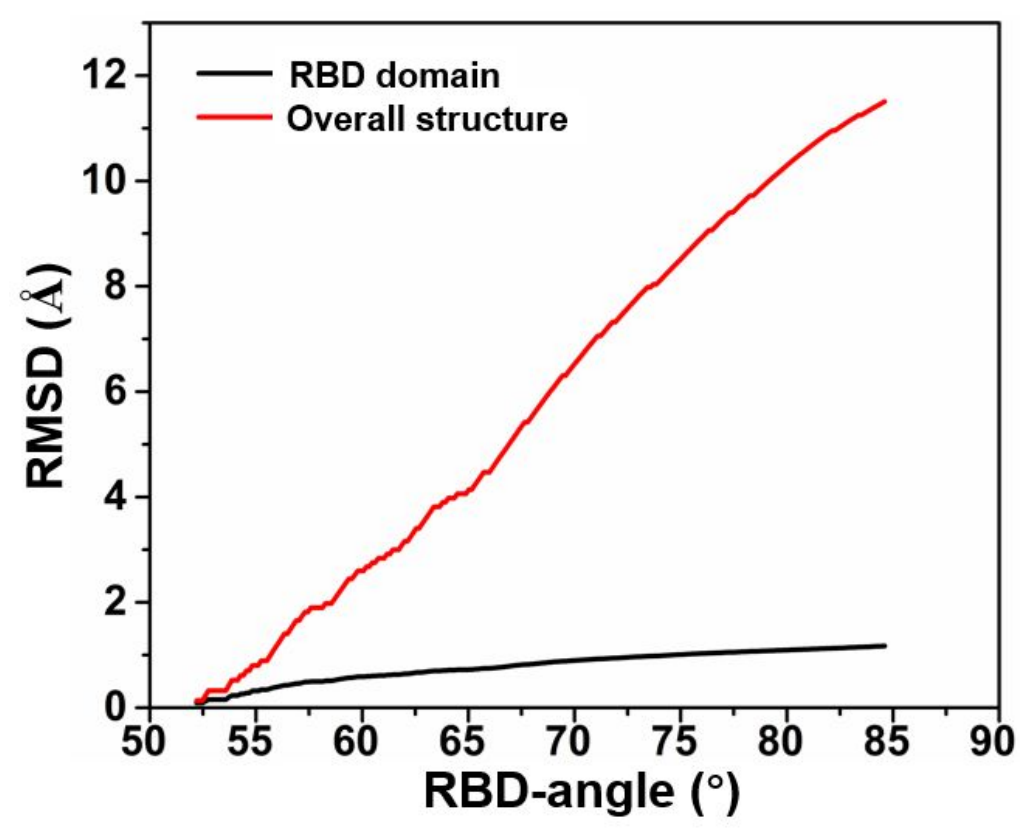

Figure S6. The RMSD values of RBD domain and overall structure along with the RBD-angle. 


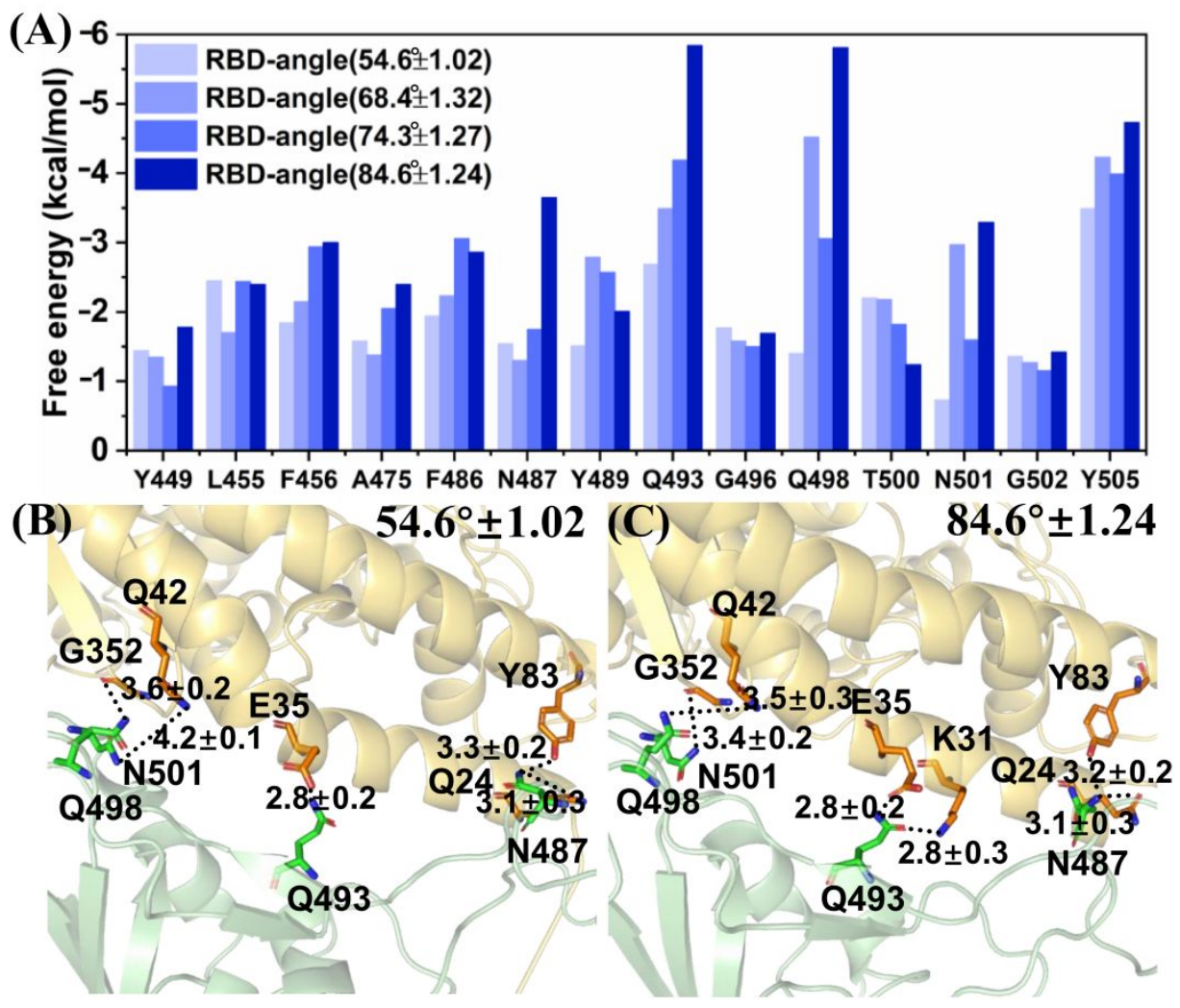

Figure S7. The ACE2-binding free energy decomposition into residues of CoV-2-S with four different RBD-angles (A). The 14 key residues of CoV-2-S was labeled. Four residues (N487, Q498, Q493, and N501, colored green) that significantly form different interactions with residues in ACE2 (colored orange) were shown in sticks for CoV-2-S with small RBD-angle (B) and large RBD-angle (C). 


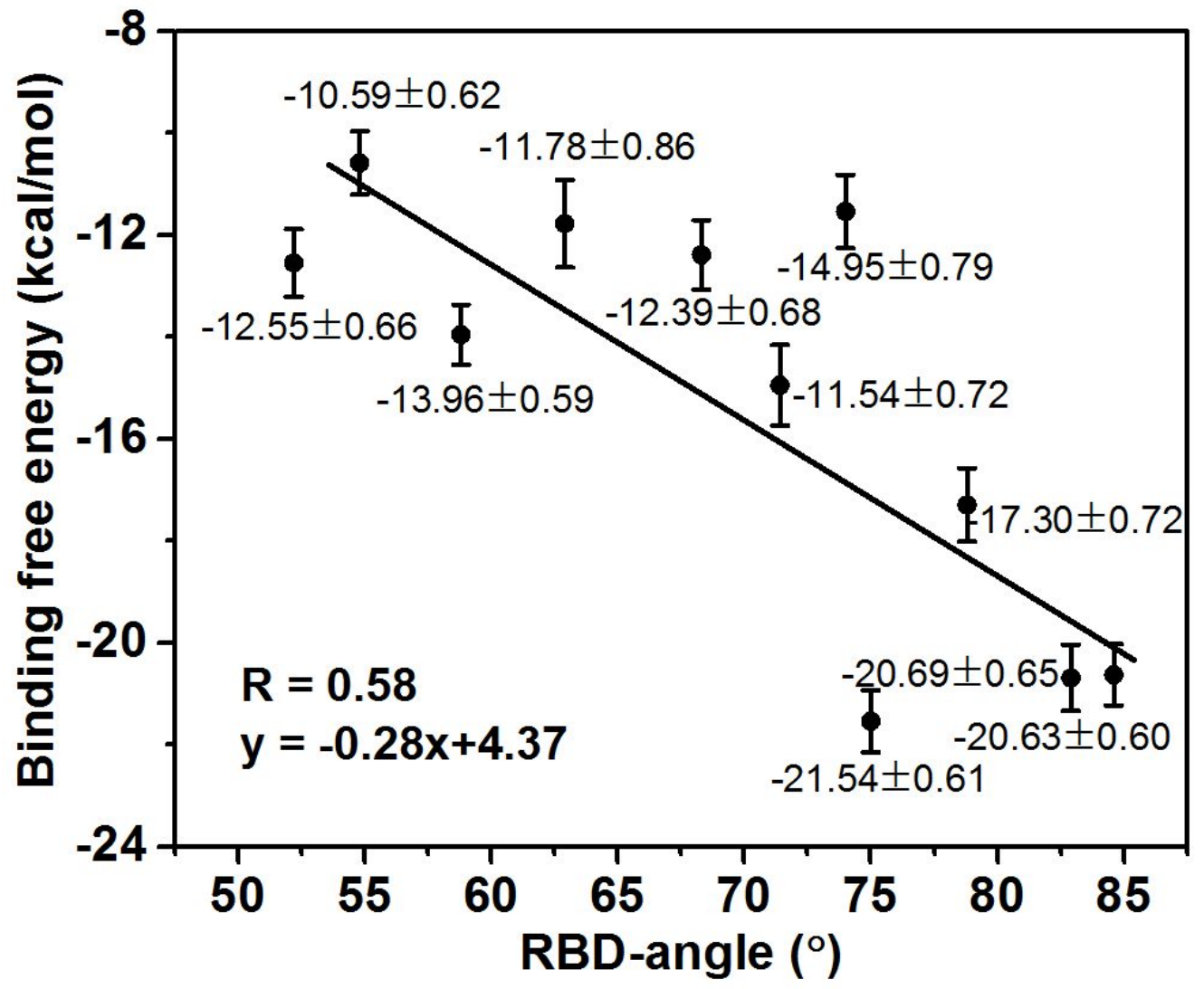

Figure S8. The calculated binding free energy of CoV-S to ACE2 against the RBDangle. The correlation coefficient $\left(R^{2}\right)$ between binding affinity and RBD-angle is 0.58 . 

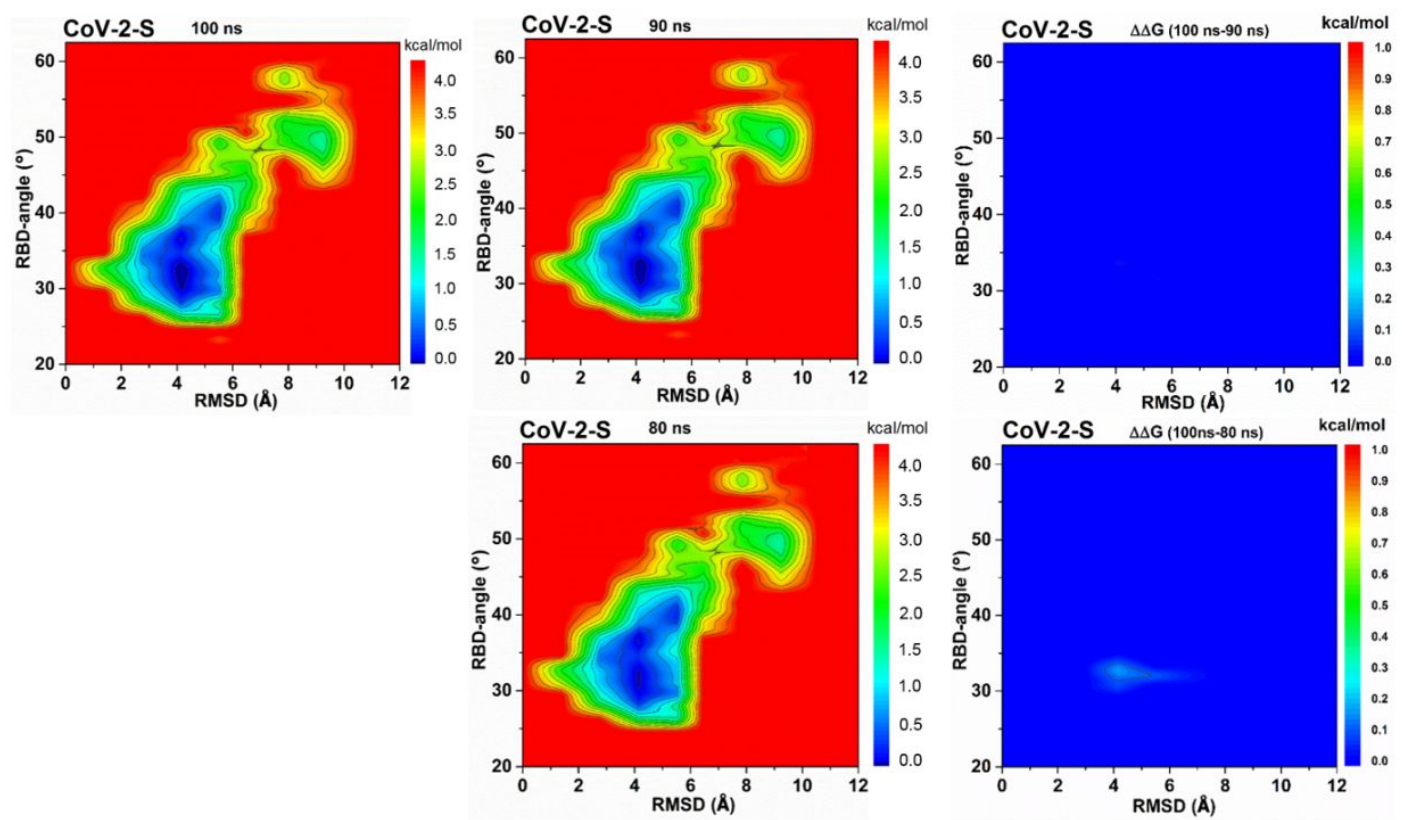

Figure S9. Free energy profiles of CoV-2-S calculated with trajectories of different simulation time $(80,90$ and $100 \mathrm{~ns})$ to evaluate the convergence of vsREMD simulations. The free energy differences $(\Delta \Delta G)$ between $100 \mathrm{~ns}$ and $90,80 \mathrm{~ns}$, respectively, were shown on the right of each column.
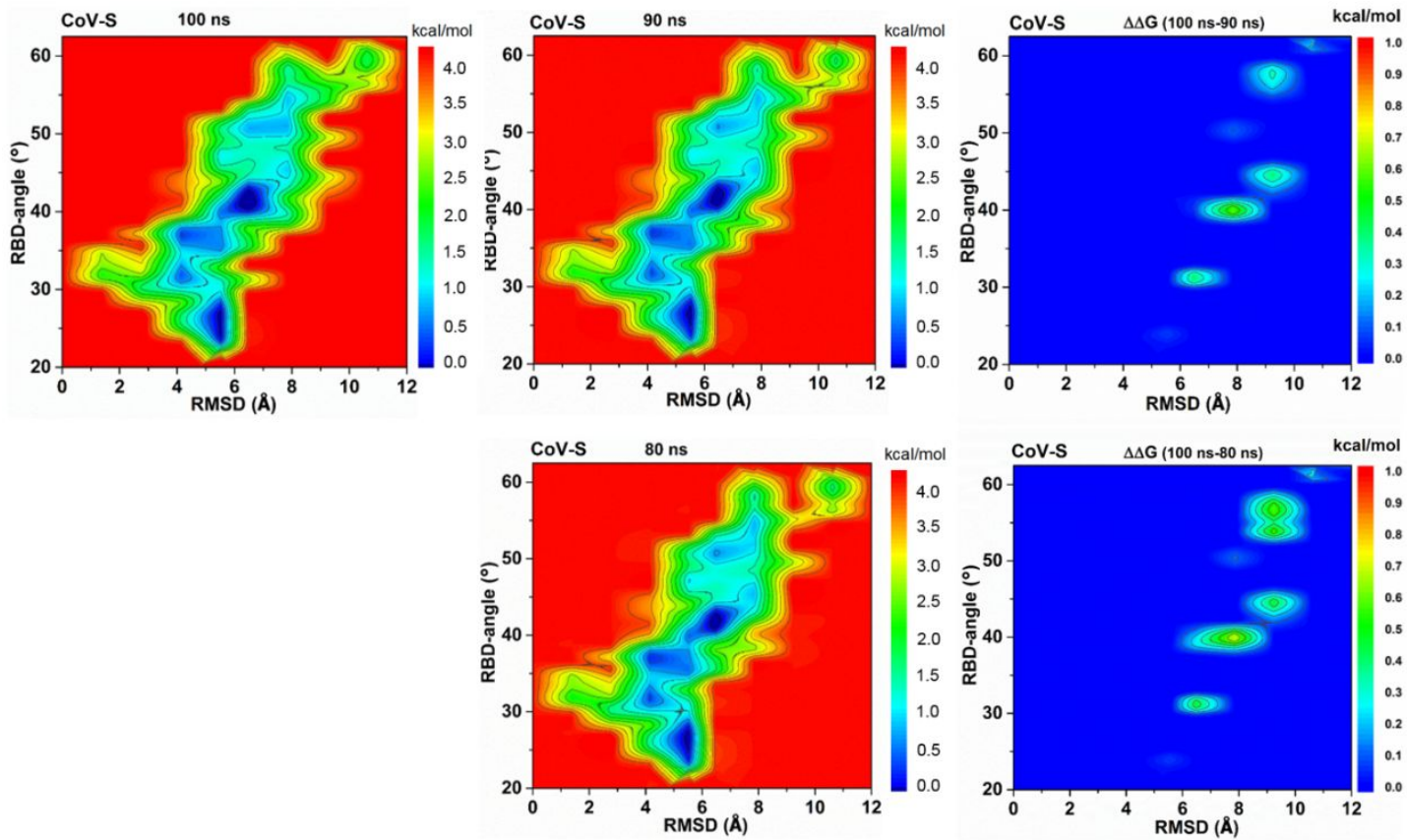

Figure S10. Free energy profiles of CoV-S calculated with trajectories of different simulation time $(80,90$ and $100 \mathrm{~ns})$ to evaluate the convergence of vsREMD simulations. The free energy differences $(\Delta \Delta G)$ between $100 \mathrm{~ns}$ and $90,80 \mathrm{~ns}$, respectively, were shown on the right of each column. 


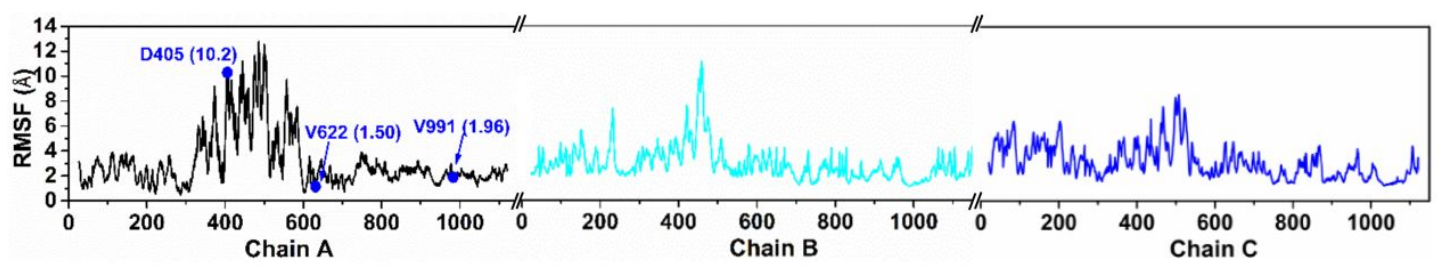

Figure S11: The root mean square fluctuation (RMSF) of each residue in COV-2-S trimer during its conformational change between the "down" and "up" states.
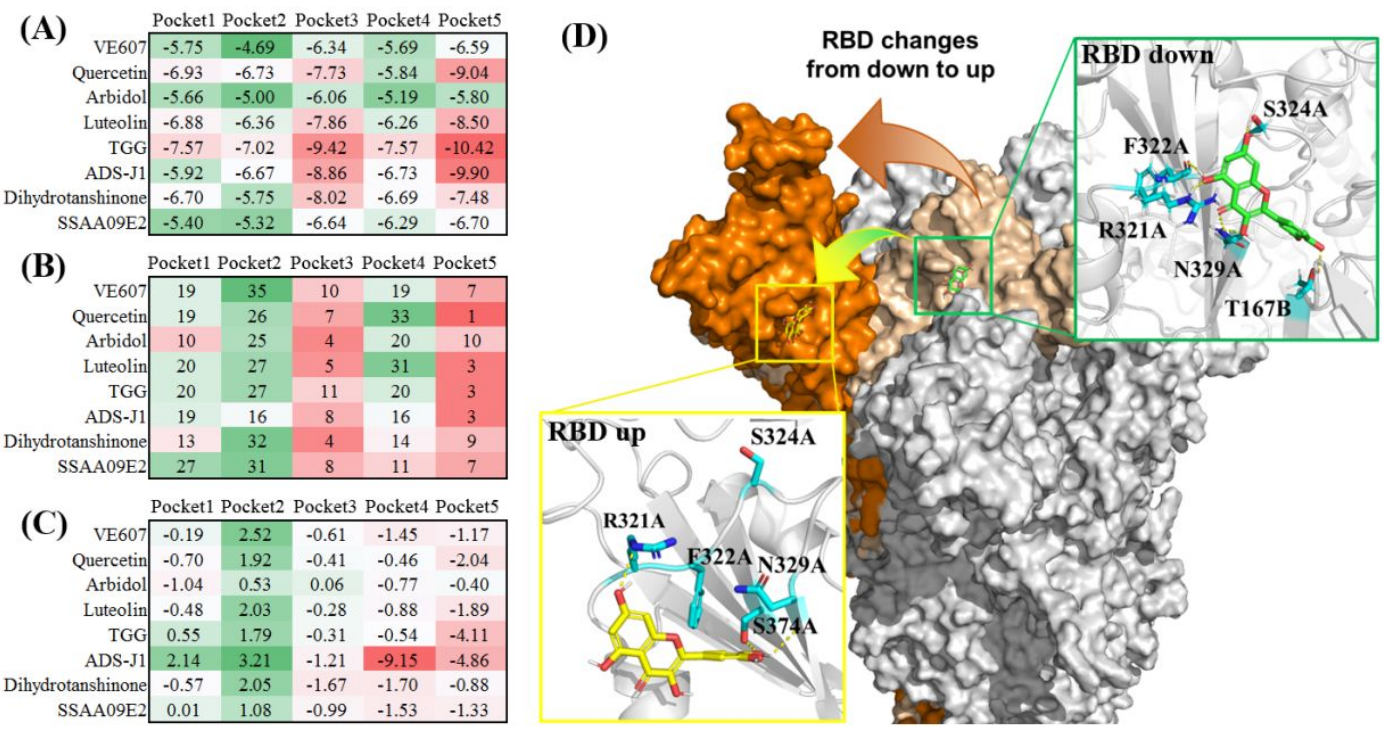

Figure S12. Molecular docking analysis of the eight compounds in the five suggested pockets by using smina software. The docking scores of the compounds for the five pockets in RBD-down CoV-2-S (A). The ranking of the eight compounds for the five pockets among 39 predicted druggable pockets by D3Pockets (B). The docking score difference between RBD-down and RBD-up CoV-2-S (C). The binding modes of quercetin in RBD-down and RBD-up CoV-2-S (D). 

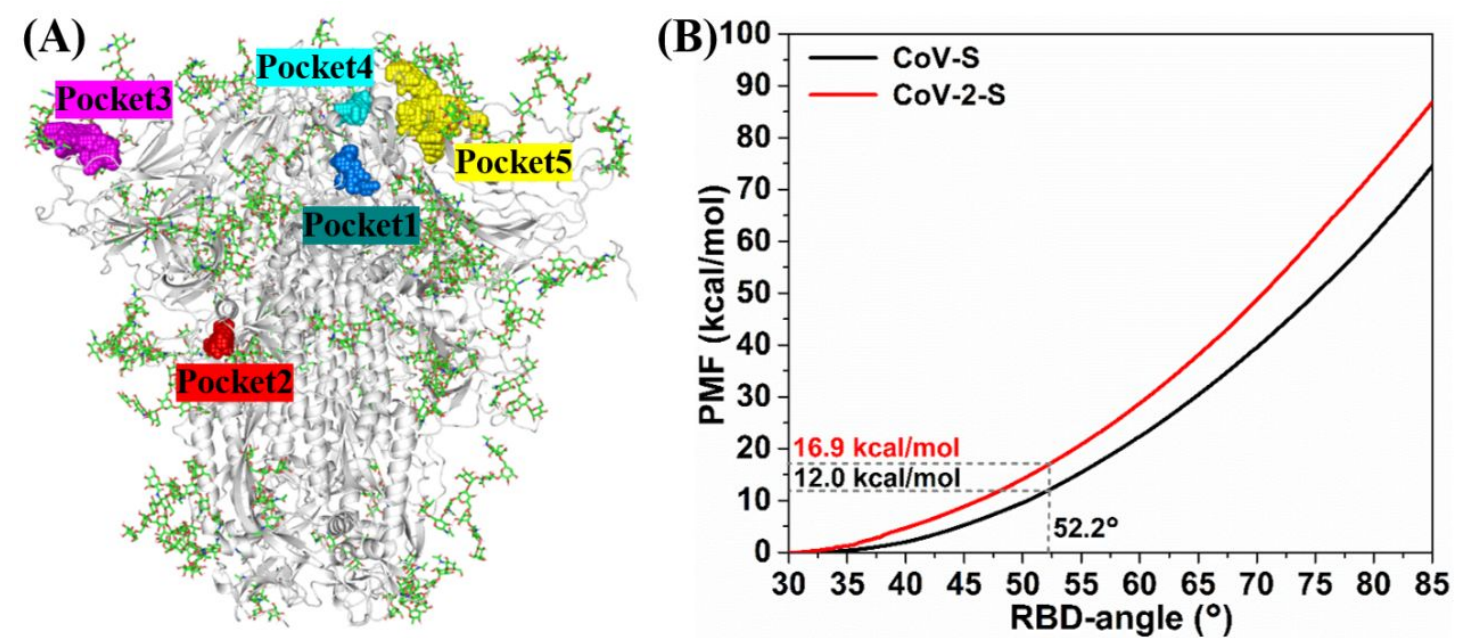

Figure S13. The five suggested pockets in the structure of glycosylated CoV-2-S (A). Glycans are shown in green sticks. The potential mean of force (PMF) of the glycosylated CoV-2-S (red) and the glycosylated CoV-S (black) (B). The PMF from RBD-angle of $30^{\circ}$ to $52.2^{\circ}$ was labeled. 

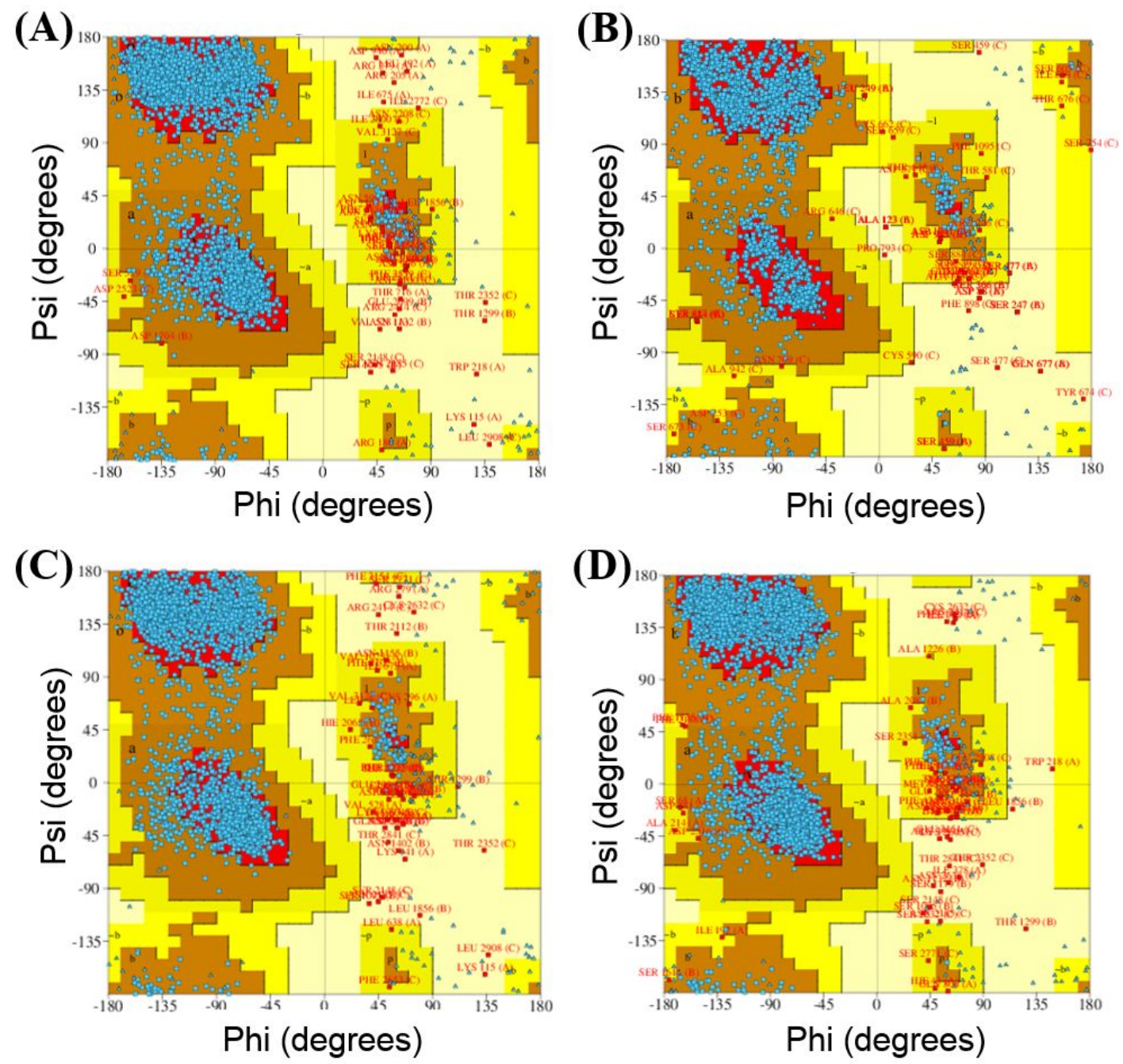

Figure S14. Ramachandran plot of the modelled structures of (A) 6ACG, (B) 6ACK, (C) 6ACJ, (D) 6CS2, calculated by PDBsum (www.ebi.ac.uk/thorntonsrv/databases/pdbsum). 


\section{Supplementary Tables}

Table S1. Summary of the CoV-S and CoV-2-S trimers in PDB.

\begin{tabular}{|c|c|c|c|c|c|c|}
\hline PDB ID & $\begin{array}{l}\text { Resolution } \\
(\AA)\end{array}$ & Organism & Chain & ACE2 & RBD state ${ }^{a}$ & RBD-angle $\left({ }^{\circ}\right)^{b}$ \\
\hline \multirow[t]{3}{*}{$6 \mathrm{X} 6 \mathrm{P}^{15}$} & 3.2 & $\mathrm{CoV}-2-\mathrm{S}$ & A & - & down & 30.9 \\
\hline & & & B & - & down & 30.2 \\
\hline & & & $\mathrm{C}$ & - & down & 31.2 \\
\hline \multirow[t]{3}{*}{$6 \mathrm{VSB}^{16}$} & 3.5 & CoV-2-S & A & - & up & - \\
\hline & & & B & - & down & - \\
\hline & & & $\mathrm{C}$ & - & -down & - \\
\hline \multirow[t]{3}{*}{$6 \mathrm{VXX}^{17}$} & 2.8 & $\mathrm{CoV}-2-\mathrm{S}$ & A & - & down & - \\
\hline & & & B & - & down & - \\
\hline & & & $\mathrm{C}$ & - & down & - \\
\hline \multirow[t]{3}{*}{$6 \mathrm{VYB}^{17}$} & 3.2 & $\mathrm{CoV}-2-\mathrm{S}$ & A & - & down & - \\
\hline & & & B & - & up & - \\
\hline & & & $\mathrm{C}$ & - & down & - \\
\hline \multirow[t]{3}{*}{$6 W_{P S}^{18}$} & 3.1 & $\mathrm{CoV}-2-\mathrm{S}$ & A & - & down & - \\
\hline & & & B & - & down & - \\
\hline & & & $\mathrm{C}$ & - & down & - \\
\hline \multirow[t]{3}{*}{$6 \mathrm{WPT}^{18}$} & 3.7 & $\mathrm{CoV}-2-\mathrm{S}$ & A & - & down & - \\
\hline & & & B & - & up & - \\
\hline & & & $\mathrm{C}$ & - & - & - \\
\hline \multirow[t]{3}{*}{$6 \times 29^{19}$} & 2.7 & CoV-2-S & A & - & down & - \\
\hline & & & B & - & down & - \\
\hline & & & $\mathrm{C}$ & - & down & - \\
\hline \multirow[t]{3}{*}{$6 \mathrm{X} 2 \mathrm{~A}^{19}$} & 3.3 & CoV-2-S & A & - & down & - \\
\hline & & & B & - & up & - \\
\hline & & & $\mathrm{C}$ & - & down & - \\
\hline \multirow[t]{3}{*}{$6 \mathrm{X} 2 \mathrm{~B}^{19}$} & 3.6 & CoV-2-S & A & - & up & - \\
\hline & & & B & - & up & - \\
\hline & & & $\mathrm{C}$ & - & up & - \\
\hline \multirow[t]{3}{*}{$6 \times 2 C^{19}$} & 3.2 & CoV-2-S & A & - & down & - \\
\hline & & & B & - & down & - \\
\hline & & & $\mathrm{C}$ & - & down & - \\
\hline $6 \mathrm{XCM}^{20}$ & 3.4 & CoV-2-S & A & - & down & - \\
\hline
\end{tabular}




\begin{tabular}{|c|c|c|c|c|c|c|}
\hline & & & B & - & up & - \\
\hline & & & $\mathrm{C}$ & - & up & - \\
\hline \multirow[t]{3}{*}{$6 \mathrm{XCN}^{20}$} & 3.7 & $\mathrm{CoV}-2-\mathrm{S}$ & A & - & up & - \\
\hline & & & B & - & up & - \\
\hline & & & $\mathrm{C}$ & - & up & - \\
\hline \multirow[t]{3}{*}{$6 \mathrm{XEY}^{21}$} & 3.2 & $\mathrm{CoV}-2-\mathrm{S}$ & A & - & down & - \\
\hline & & & B & - & down & - \\
\hline & & & $\mathrm{C}$ & - & down & - \\
\hline \multirow[t]{3}{*}{$6 \mathrm{XKL}^{22}$} & 3.2 & $\mathrm{CoV}-2-\mathrm{S}$ & A & - & up & - \\
\hline & & & B & - & down & - \\
\hline & & & $\mathrm{C}$ & - & down & - \\
\hline \multirow[t]{3}{*}{$6 \mathrm{XM} 5^{23}$} & 3.1 & $\mathrm{CoV}-2-\mathrm{S}$ & A & - & down & 31.1 \\
\hline & & & B & - & down & 30.7 \\
\hline & & & $\mathrm{C}$ & - & down & 31.0 \\
\hline \multirow[t]{3}{*}{$6 \mathrm{XR} 8^{24}$} & 2.9 & CoV-2-S & A & - & down & - \\
\hline & & & B & - & down & - \\
\hline & & & $\mathrm{C}$ & - & down & - \\
\hline \multirow[t]{3}{*}{$6 \mathrm{XS}^{25}$} & 3.7 & CoV-2-S & A & - & - & - \\
\hline & & & B & - & - & - \\
\hline & & & $\mathrm{C}$ & - & - & - \\
\hline \multirow[t]{3}{*}{$6 Z 43^{26}$} & 3.3 & CoV-2-S & A & - & up & - \\
\hline & & & B & - & up & - \\
\hline & & & $\mathrm{C}$ & - & up & - \\
\hline \multirow[t]{3}{*}{$6 Z 97^{27}$} & 3.4 & $\mathrm{CoV}-2-\mathrm{S}$ & A & - & down & - \\
\hline & & & B & - & up & - \\
\hline & & & $\mathrm{C}$ & - & down & - \\
\hline \multirow[t]{3}{*}{$6 \mathrm{ZDH}^{26}$} & 3.7 & CoV-2-S & A & - & up & - \\
\hline & & & B & - & up & - \\
\hline & & & $\mathrm{C}$ & - & up & - \\
\hline \multirow[t]{3}{*}{$6 \mathrm{ZGE}^{28}$} & 2.6 & CoV-2-S & A & - & down & - \\
\hline & & & B & - & down & - \\
\hline & & & $\mathrm{C}$ & - & down & - \\
\hline \multirow[t]{3}{*}{$6 \mathrm{ZGG}^{28}$} & 3.8 & CoV-2-S & A & - & down & - \\
\hline & & & B & - & up & - \\
\hline & & & $\mathrm{C}$ & - & down & - \\
\hline $6 \mathrm{ZGH}^{28}$ & 6.8 & $\mathrm{CoV}-2-\mathrm{S}$ & A & - & - & - \\
\hline
\end{tabular}




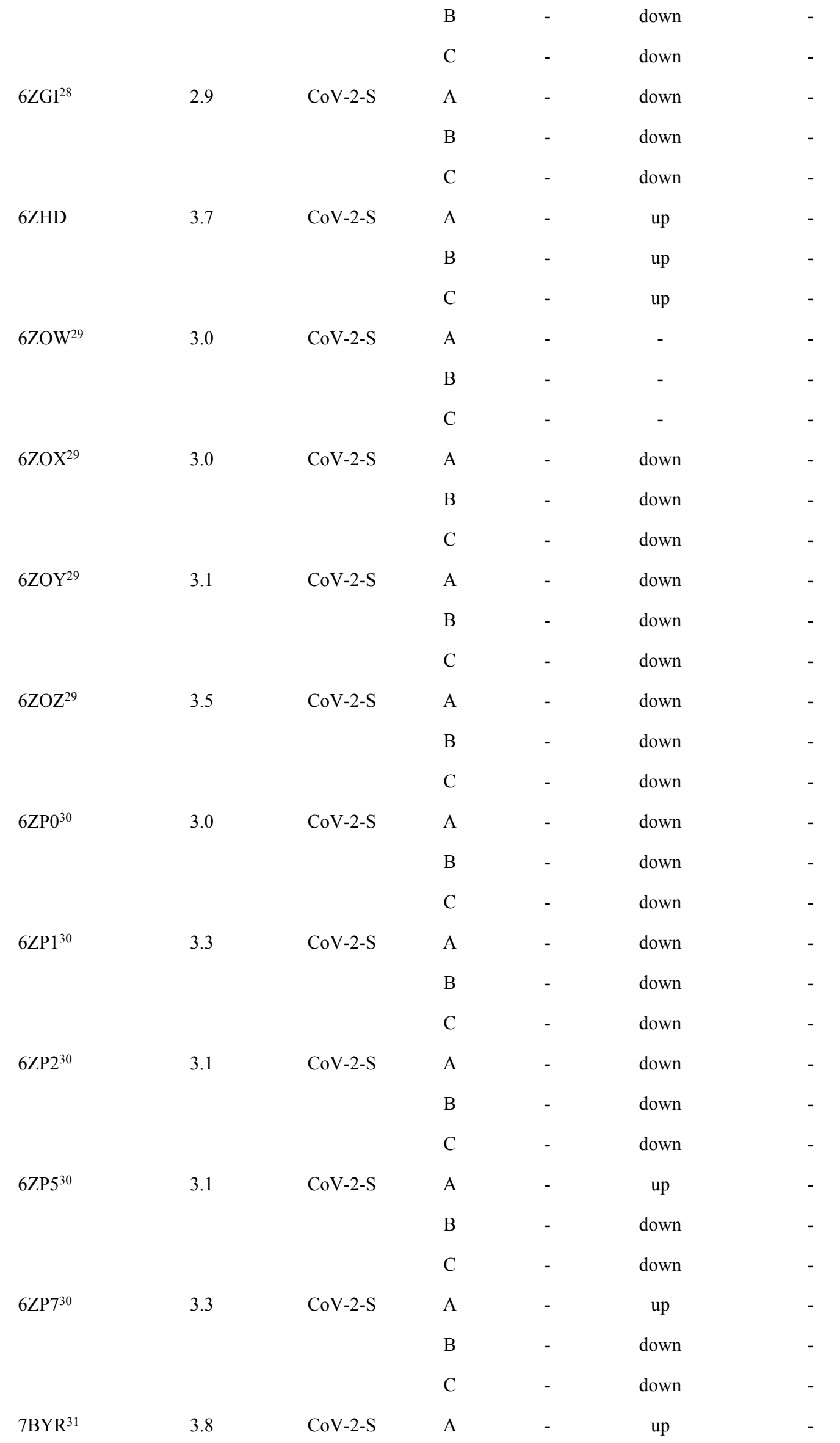




\begin{tabular}{|c|c|c|c|c|c|c|}
\hline & & & B & - & up & - \\
\hline & & & $\mathrm{C}$ & - & up & - \\
\hline $7 \mathrm{C} 2 \mathrm{~L}^{32}$ & 3.1 & $\mathrm{CoV}-2-\mathrm{S}$ & A & - & up & - \\
\hline & & & B & - & - & - \\
\hline & & & $\mathrm{C}$ & - & - & - \\
\hline $5 \mathrm{WRG}^{33}$ & 4.3 & $\mathrm{CoV}-\mathrm{S}$ & A & - & down & 30.1 \\
\hline & & & B & - & down & 30.1 \\
\hline & & & $\mathrm{C}$ & - & down & 30.1 \\
\hline $5 \times 58^{13}$ & 3.2 & CoV-S & A & - & down & 31.6 \\
\hline & & & B & - & down & 31.6 \\
\hline & & & $\mathrm{C}$ & - & down & 30.7 \\
\hline $5 \mathrm{X} \mathrm{B}^{13}$ & 3.7 & CoV-S & A & - & up & 84.8 \\
\hline & & & B & - & down & 30.9 \\
\hline & & & $\mathrm{C}$ & - & down & 30.9 \\
\hline $5 \mathrm{XLR}^{33}$ & 3.8 & CoV-S & A & - & down & 32.1 \\
\hline & & & B & - & down & 32.1 \\
\hline & & & $\mathrm{C}$ & - & down & 32.1 \\
\hline $6 \mathrm{ACC}^{2}$ & 3.6 & CoV-S & A & - & down & 33.4 \\
\hline & & & B & - & down & 33.4 \\
\hline & & & $\mathrm{C}$ & - & down & 33.4 \\
\hline $6 \mathrm{ACD}^{2}$ & 3.9 & CoV-S & A & - & down & 32.8 \\
\hline & & $-S$ & B & - & down & 32.8 \\
\hline & & & $\mathrm{C}$ & - & down & 32.9 \\
\hline $6 \mathrm{ACG}^{2}$ & 5.4 & CoV-S & A & - & down & 32.6 \\
\hline & & & B & - & down & 32.7 \\
\hline & & & $\mathrm{C}$ & ACE2 & up & 54.8 \\
\hline $6 \mathrm{ACJ}^{2}$ & 4.2 & CoV-S & A & - & down & 33.0 \\
\hline & & & B & - & down & 33.3 \\
\hline & & & C & ACE2 & up & 68.3 \\
\hline $6 \mathrm{ACK}^{2}$ & 4.5 & CoV-S & A & - & down & 33.1 \\
\hline & & & B & - & down & 33.8 \\
\hline & & & $\mathrm{C}$ & ACE2 & up & 84.6 \\
\hline $6 \mathrm{CRV}^{3}$ & 3.2 & CoV-S & A & - & - & - \\
\hline & & & B & - & - & - \\
\hline & & & C & - & - & - \\
\hline $6 \mathrm{CRW}^{3}$ & 3.9 & CoV-S & A & - & down & 34.3 \\
\hline
\end{tabular}




\begin{tabular}{|c|c|c|c|c|c|c|}
\hline & & & B & - & up & 68.8 \\
\hline & & & $\mathrm{C}$ & - & down & 34.2 \\
\hline \multirow[t]{3}{*}{$6 \mathrm{CRX} \mathrm{X}^{3}$} & 3.9 & CoV-S & A & - & up & 71.6 \\
\hline & & & B & - & up & 70.6 \\
\hline & & & C & - & down & 38.1 \\
\hline \multirow[t]{3}{*}{$6 \mathrm{CRZ}^{3}$} & 3.3 & CoV-S & A & - & down & 34.1 \\
\hline & & & B & - & up & 68.8 \\
\hline & & & $\mathrm{C}$ & - & down & 34.1 \\
\hline \multirow[t]{3}{*}{$6 \mathrm{CS} 0^{3}$} & 3.8 & CoV-S & A & - & down & 34.2 \\
\hline & & & B & - & up & 68.8 \\
\hline & & & C & - & down & 34.1 \\
\hline \multirow[t]{3}{*}{$6 \mathrm{CS}^{3}$} & 4.6 & CoV-S & A & - & up & 71.6 \\
\hline & & & B & - & up & 70.7 \\
\hline & & & $\mathrm{C}$ & - & down & 38.1 \\
\hline \multirow[t]{3}{*}{$6 \mathrm{CS} 2^{3}$} & 4.4 & CoV-S & A & - & - & - \\
\hline & & & B & ACE2 & up & 74.0 \\
\hline & & & $\mathrm{C}$ & - & - & - \\
\hline \multirow[t]{3}{*}{$6 \mathrm{NB}^{34}$} & 4.2 & CoV-S & A & - & down & 30.7 \\
\hline & & & B & - & up & 77.9 \\
\hline & & & $\mathrm{C}$ & - & up & 55.2 \\
\hline \multirow[t]{3}{*}{$6 \mathrm{NB} 7^{34}$} & 4.5 & CoV-S & A & - & up & 75.3 \\
\hline & & & B & - & up & 70.6 \\
\hline & & & $\mathrm{C}$ & - & up & 78.5 \\
\hline
\end{tabular}

a: The RBD state is assigned according to the corresponding references.

b: The RBD-angle is determined by the residues D392-T608-V973 in the CoV-S, and D405V622-V991 in the CoV-2-S. 
Table S2. Components of the predicted binding free energy $(\mathrm{kcal} / \mathrm{mol})$ for $\mathrm{CoV}-\mathrm{S}$ and CoV-2-S trimers binding to ACE2 by using the MM/GBSA method ${ }^{\mathrm{a}}$

\begin{tabular}{lllll}
\hline & $\mathbf{6 A C G}$ & $\mathbf{6 A C J}$ & $\mathbf{6 C S 2}$ & $\mathbf{6 A C K}$ \\
\hline RBD-angle & $54.1^{\circ} \pm 1.24^{\mathrm{c}}$ & $68.0^{\circ} \pm 1.19$ & $74.5^{\circ} \pm 0.98$ & $84.4^{\circ} \pm 1.49$ \\
$\mathbf{C o V}-\mathbf{S}$ & & & & \\
$E_{v d w}$ & $-74.67 \pm 0.60$ & $-84.99 \pm 0.54$ & $-81.48 \pm 0.69$ & $-86.15 \pm 0.47$ \\
$E_{e l e}$ & $18.69 \pm 0.66$ & $-53.59 \pm 0.56$ & $-109.67 \pm 3.05$ & $-120.10 \pm 3.29$ \\
$E_{g b}$ & $34.91 \pm 0.82$ & $122.73 \pm 0.68$ & $173.18 \pm 3.09$ & $182.77 \pm 3.26$ \\
$E_{n p}$ & $-9.72 \pm 0.08$ & $-11.10 \pm 0.07$ & $-10.42 \pm 0.10$ & $-12.04 \pm 0.06$ \\
$\Delta H$ & $-24.79 \pm 0.61$ & $-26.96 \pm 0.56$ & $-28.39 \pm 0.70$ & $-35.52 \pm 0.54$ \\
$-\mathrm{T} \Delta \mathrm{S}$ & $-14.20 \pm 0.62$ & $-14.57 \pm 0.80$ & $-16.85 \pm 0.74$ & $-14.89 \pm 0.67$ \\
$\Delta \mathrm{G}$ & $-10.59 \pm 0.62$ & $-12.39 \pm 0.68$ & $-11.54 \pm 0.72$ & $-20.63 \pm 0.60$ \\
$\mathbf{C o V}-2-\mathbf{S}^{\mathbf{b}}$ & $54.6^{\circ} \pm 1.02$ & $68.4^{\circ} \pm 1.32$ & $74.3^{\circ} \pm 1.27$ & $84.6^{\circ} \pm 1.24$ \\
$E_{v d w}$ & $-81.34 \pm 0.47$ & $-95.90 \pm 0.53$ & $-100.86 \pm 0.72$ & $-106.25 \pm 0.56$ \\
$E_{e l e}$ & $-816.09 \pm 0.59$ & $-796.20 \pm 2.59$ & $-763.73 \pm 3.59$ & $-763.10 \pm 2.86$ \\
$E_{g b}$ & $875.10 \pm 0.52$ & $866.54 \pm 2.59$ & $830.25 \pm 3.43$ & $828.67 \pm 2.66$ \\
$E_{n p}$ & $-11.14 \pm 0.07$ & $-13.98 \pm 0.06$ & $-14.61 \pm 0.08$ & $-15.21 \pm 0.05$ \\
$\Delta H$ & $-33.47 \pm 0.71$ & $-39.55 \pm 0.56$ & $-48.95 \pm 0.95$ & $-55.89 \pm 0.56$ \\
$-\mathrm{T} \Delta \mathrm{S}$ & $-15.47 \pm 0.98$ & $-14.45 \pm 0.81$ & $-16.55 \pm 0.78$ & $-16.37 \pm 0.66$ \\
$\Delta \mathrm{G}$ & $-18.00 \pm 0.84$ & $-25.10 \pm 0.68$ & $-32.40 \pm 0.86$ & $-39.52 \pm 0.61$ \\
\hline
\end{tabular}

a: The statistical error was estimated based on 50-100 ns MD simulation trajectory. 1000 snapshots evenly extracted from the 50-100 ns MD trajectory of each complex were used for MM/GBSA calculations and 10 snapshots for the entropy term calculations.

b: The three-dimensional (3D) models of ACE2-bound CoV-2-S trimer was modelled taking the four $\mathrm{CoV}-\mathrm{S}$ trimers as templates.

$\mathrm{c}$ : The average and standard deviation of RBD-angle during simulations. 
Table S3. Residues of CoV-S in RBD-ACE2 artificial interface.*

\begin{tabular}{l|l}
\hline Same to SARS-CoV-2-S & Mutated residues (wide type-mutation) \\
\hline Y436; Y440; P470; A471; & K390-R390; R426-N426; S432-V432; T433- \\
N473; Y475; Y481; G482; & G433; Y442-L442; L443-F443; F460-Y460; \\
F483; T486; G488; G490; & P462-A462; D463-G463; K465-T465; L472- \\
Y491; Q492. & F472; Y476-F476; N479-Q479; D480-S480; \\
& Y484-Q484; T485-P485; T487-N487; I489- \\
& V489 \\
\hline
\end{tabular}

*: the residues were determined according to the distance between the RBD and ACE2 less than 7 $\AA$. 
Table S4. Compounds targeting spike protein to block the entry of coronavirus into host cells.

\begin{tabular}{|c|c|c|c|}
\hline Name & Structure & Organism & Activity \\
\hline VE60735 & & SARS-CoV & $\begin{array}{c}\mathrm{EC}_{50}=1.6 \\
\mu \mathrm{M}\end{array}$ \\
\hline Quercetin $^{36}$ & & SARS-CoV & $\begin{array}{c}\mathrm{IC}_{50}=83.4 \\
\mu \mathrm{M}\end{array}$ \\
\hline Arbidol $^{37}$ & & SARS-CoV-2 & $\begin{array}{c}\mathrm{EC}_{50}=4.11 \\
\mu \mathrm{M}\end{array}$ \\
\hline Luteolin $^{36}$ & & SARS-CoV & $\begin{array}{c}\mathrm{IC}_{50}=10.6 \\
\mu \mathrm{M}\end{array}$ \\
\hline $\mathrm{TGG}^{36}$ & & SARS-CoV & $\begin{array}{c}\mathrm{EC}_{50}=4.5 \\
\mu \mathrm{M}\end{array}$ \\
\hline ADS-J138 & & MERS-CoV & $\begin{array}{c}\mathrm{IC}_{50}=0.6 \\
\mu \mathrm{M}\end{array}$ \\
\hline Dihydrotanshinone $^{39}$ & & MERS-CoV & / \\
\hline SSAA09E2 $2^{40}$ & & SARS-CoV & $\begin{array}{c}\mathrm{EC}_{50}=3.1 \\
\mu \mathrm{M}\end{array}$ \\
\hline
\end{tabular}


Table S5. The glycosylation of CoV-2-S according to experimental data ${ }^{41-42}$.

\begin{tabular}{|c|c|c|}
\hline Residues & Type & Glycan \\
\hline N61A & M5 & $\operatorname{aDMan}(1 \rightarrow 6)[\operatorname{aDMan}(1 \rightarrow 3)] \mathrm{aDMan}(1 \rightarrow 6)[\operatorname{aDMan}(1 \rightarrow 3)] \mathrm{bDMan}(1 \rightarrow 4) \mathrm{bDGlcNAc}(1 \rightarrow 4) \mathrm{bDGlcNAc}(1 \rightarrow) \mathrm{N} 61$ \\
\hline N74A & A3 & $\begin{array}{l}\operatorname{bDGlcNAc}(1 \rightarrow 6)[\mathrm{bDGlcNAc}(1 \rightarrow 2)] \mathrm{aDMan}(1 \rightarrow 6)[\mathrm{bDGlcNAc}(1 \rightarrow 2) \mathrm{aDMan}(1 \rightarrow 3)] \mathrm{bDMan}(1 \rightarrow 4) \mathrm{bDGlcNAc}(1 \rightarrow \\
\text { 4)bDGlcNAc}(1 \rightarrow) \mathrm{N} 74\end{array}$ \\
\hline N122A & M5 & $\operatorname{aDMan}(1 \rightarrow 6)[\operatorname{aDMan}(1 \rightarrow 3)] \mathrm{aDMan}(1 \rightarrow 6)[\operatorname{aDMan}(1 \rightarrow 3)] \mathrm{bDMan}(1 \rightarrow 4) \mathrm{bDGlcNAc}(1 \rightarrow 4) \mathrm{bDGlcNAc}(1 \rightarrow) \mathrm{N} 122$ \\
\hline N149A & FA2G2S1 & $\begin{array}{l}\mathrm{aDNeu} 5 \operatorname{Ac}(2 \rightarrow 6) \mathrm{bDGal}(1 \rightarrow 4) \mathrm{bDGlcNAc}(1 \rightarrow 2) \mathrm{aDMan}(1 \rightarrow 6)[\mathrm{bDGal}(1 \rightarrow 4) \mathrm{bDGlcNAc}(1 \rightarrow 2) \mathrm{aDMan}(1 \rightarrow \\
\text { 3)]bDMan }(1 \rightarrow 4) \mathrm{bDGlcNAc}(1 \rightarrow 4)[\operatorname{aLFuc}(1 \rightarrow 6)] \mathrm{bDGlcNAc}(1 \rightarrow) \mathrm{N} 149\end{array}$ \\
\hline N165A & FA2G2S2 & $\begin{array}{l}\text { xaDNeu5Ac }(2 \rightarrow 6) \mathrm{bDGal}(1 \rightarrow 4) \mathrm{bDGlcNAc}(1 \rightarrow 2) \mathrm{aDMan}(1 \rightarrow 6)[\operatorname{aDNeu} 5 \mathrm{Ac}(2 \rightarrow 6) \mathrm{bDGal}(1 \rightarrow 4) \mathrm{bDGlcNAc}(1 \rightarrow \\
\text { 2)aDMan }(1 \rightarrow 3)] \mathrm{bDMan}(1 \rightarrow 4) \mathrm{bDGlcNAc}(1 \rightarrow 4)[\operatorname{aLFuc}(1 \rightarrow 6)] \mathrm{bDGlcNAc}(1 \rightarrow) \mathrm{N} 165\end{array}$ \\
\hline $\mathrm{N} 234 \mathrm{~A}$ & M8 & $\begin{array}{l}\operatorname{aDMan}(1 \rightarrow 2) \mathrm{aDMan}(1 \rightarrow 6)[\operatorname{aDMan}(1 \rightarrow 3)] \mathrm{aDMan}(1 \rightarrow 6)[\operatorname{aDMan}(1 \rightarrow 2) \operatorname{aDMan}(1 \rightarrow 2) \mathrm{aDMan}(1 \rightarrow 3)] \mathrm{bDMan}(1 \rightarrow \\
\text { 4)bDGlcNAc}(1 \rightarrow 4) \mathrm{bDGlcNAc}(1 \rightarrow) \mathrm{N} 234\end{array}$ \\
\hline N282A & FA3 & $\begin{array}{l}\operatorname{bDGlcNAc}(1 \rightarrow 6)[\mathrm{bDGlcNAc}(1 \rightarrow 2)] \mathrm{aDMan}(1 \rightarrow 6)[\mathrm{bDGlcNAc}(1 \rightarrow 2) \mathrm{aDMan}(1 \rightarrow 3)] \mathrm{bDMan}(1 \rightarrow 4) \mathrm{bDGlcNAc}(1 \rightarrow \\
\text { 4) }[\operatorname{aLFuc}(1 \rightarrow 6)] \mathrm{bDGlcNAc}(1 \rightarrow) \mathrm{N} 282\end{array}$ \\
\hline N331A & FA2 & $\begin{array}{l}\operatorname{bDGlcNAc}(1 \rightarrow 2) \mathrm{aDMan}(1 \rightarrow 6)[\mathrm{bDGlcNAc}(1 \rightarrow 2) \operatorname{aDMan}(1 \rightarrow 3)] \mathrm{bDMan}(1 \rightarrow 4) \operatorname{bDGlcNAc}(1 \rightarrow 4)[\operatorname{aLFuc}(1 \rightarrow \\
\text { 6)]bDGlcNAc}(1 \rightarrow) \mathrm{N} 331\end{array}$ \\
\hline N343A & FA2 & $\begin{array}{l}\operatorname{bDGlcNAc}(1 \rightarrow 2) \mathrm{aDMan}(1 \rightarrow 6)[\mathrm{bDGlcNAc}(1 \rightarrow 2) \mathrm{aDMan}(1 \rightarrow 3)] \mathrm{bDMan}(1 \rightarrow 4) \mathrm{bDGlcNAc}(1 \rightarrow 4)[\operatorname{aLFuc}(1 \rightarrow \\
\text { 6)]bDGlcNAc}(1 \rightarrow) \mathrm{N} 343\end{array}$ \\
\hline N603A & FA2 & $\begin{array}{l}\operatorname{bDGlcNAc}(1 \rightarrow 2) \mathrm{aDMan}(1 \rightarrow 6)[\mathrm{bDGlcNAc}(1 \rightarrow 2) \mathrm{aDMan}(1 \rightarrow 3)] \mathrm{bDMan}(1 \rightarrow 4) \operatorname{bDGlcNAc}(1 \rightarrow 4)[\operatorname{aLFuc}(1 \rightarrow \\
\text { 6)]bDGlcNAc}(1 \rightarrow) \mathrm{N} 603\end{array}$ \\
\hline N616A & A2 & $\begin{array}{l}\operatorname{bDGlcNAc}(1 \rightarrow 2) \mathrm{aDMan}(1 \rightarrow 6)[\mathrm{bDGlcNAc}(1 \rightarrow 2) \mathrm{aDMan}(1 \rightarrow 3)] \mathrm{bDMan}(1 \rightarrow 4) \mathrm{bDGlcNAc}(1 \rightarrow 4) \mathrm{bDGlcNAc}(1 \\
\rightarrow) \mathrm{N} 616\end{array}$ \\
\hline N657A & M5 & $\operatorname{aDMan}(1 \rightarrow 6)[\operatorname{aDMan}(1 \rightarrow 3)] \mathrm{aDMan}(1 \rightarrow 6)[\operatorname{aDMan}(1 \rightarrow 3)] \mathrm{bDMan}(1 \rightarrow 4) \mathrm{bDGlcNAc}(1 \rightarrow 4) \mathrm{bDGlcNAc}(1 \rightarrow) \mathrm{N} 657$ \\
\hline N709A & M6 & $\begin{array}{l}\operatorname{aDMan}(1 \rightarrow 6)[\operatorname{aDMan}(1 \rightarrow 3)] \mathrm{aDMan}(1 \rightarrow 6)[\mathrm{aDMan}(1 \rightarrow 2) \mathrm{aDMan}(1 \rightarrow 3)] \mathrm{bDMan}(1 \rightarrow 4) \mathrm{bDGlcNAc}(1 \rightarrow \\
\text { 4)bDGlcNAc}(1 \rightarrow) \mathrm{N} 709\end{array}$ \\
\hline N717A & Hydrid G1 & $\begin{array}{l}\operatorname{bDGal}(1 \rightarrow 4) \mathrm{bDGlcNAc}(1 \rightarrow 2) \mathrm{aDMan}(1 \rightarrow 3)[\mathrm{aDMan}(1 \rightarrow 6)[\mathrm{aDMan}(1 \rightarrow 3)] \mathrm{aDMan}(1 \rightarrow 6)] \mathrm{bDMan}(1 \rightarrow \\
\text { 4)bDGlcNAc}(1 \rightarrow 4) \mathrm{bDGlcNAc}(1 \rightarrow) \mathrm{N} 717\end{array}$ \\
\hline N801A & M6 & $\begin{array}{l}\operatorname{aDMan}(1 \rightarrow 6)[\operatorname{aDMan}(1 \rightarrow 3)] \mathrm{aDMan}(1 \rightarrow 6)[\mathrm{aDMan}(1 \rightarrow 2) \mathrm{aDMan}(1 \rightarrow 3)] \mathrm{bDMan}(1 \rightarrow 4) \mathrm{bDGlcNAc}(1 \rightarrow \\
\text { 4)bDGlcNAc}(1 \rightarrow) \mathrm{N} 801\end{array}$ \\
\hline N1074A & FA2G2S1 & $\begin{array}{l}\mathrm{aDNeu} 5 \operatorname{Ac}(2 \rightarrow 6) \mathrm{bDGal}(1 \rightarrow 4) \mathrm{bDGlcNAc}(1 \rightarrow 2) \mathrm{aDMan}(1 \rightarrow 3)[\mathrm{bDGal}(1 \rightarrow 4) \mathrm{bDGlcNAc}(1 \rightarrow 2) \mathrm{aDMan}(1 \rightarrow \\
\text { 6)]bDMan }(1 \rightarrow 4) \mathrm{bDGlcNAc}(1 \rightarrow 4)[\operatorname{aLFuc}(1 \rightarrow 6)] \mathrm{bDGlcNAc}(1 \rightarrow) \mathrm{N} 1074\end{array}$ \\
\hline N1098A & FA2 & $\begin{array}{l}\operatorname{bDGlcNAc}(1 \rightarrow 2) \mathrm{aDMan}(1 \rightarrow 6)[\mathrm{bDGlcNAc}(1 \rightarrow 2) \mathrm{aDMan}(1 \rightarrow 3)] \mathrm{bDMan}(1 \rightarrow 4) \mathrm{bDGlcNAc}(1 \rightarrow 4)[\operatorname{aLFuc}(1 \rightarrow \\
\text { 6)]bDGlcNAc}(1 \rightarrow) \mathrm{N} 1098\end{array}$ \\
\hline N61B & M5 & $\operatorname{aDMan}(1 \rightarrow 6)[\operatorname{aDMan}(1 \rightarrow 3)] \mathrm{aDMan}(1 \rightarrow 6)[\operatorname{aDMan}(1 \rightarrow 3)] \mathrm{bDMan}(1 \rightarrow 4) \mathrm{bDGlcNAc}(1 \rightarrow 4) \mathrm{bDGlcNAc}(1 \rightarrow) \mathrm{N} 61$ \\
\hline N74B & FA3G3S2 & $\begin{array}{l}\mathrm{aDNeu} 5 \mathrm{Ac}(2 \rightarrow 6) \mathrm{bDGal}(1 \rightarrow 4) \mathrm{bDGlcNAc}(1 \rightarrow 6)[\mathrm{bDGal}(1 \rightarrow 4) \mathrm{bDGlcNAc}(1 \rightarrow 2)] \operatorname{aDMan}(1 \rightarrow 6)[\operatorname{aDNeu} 5 \operatorname{Ac}(2 \rightarrow \\
6) \mathrm{bDGal}(1 \rightarrow 4) \mathrm{bDGlcNAc}(1 \rightarrow 2) \mathrm{aDMan}(1 \rightarrow 3)] \mathrm{bDMan}(1 \rightarrow 4) \mathrm{bDGlcNAc}(1 \rightarrow 4)[\operatorname{aLFuc}(1 \rightarrow 6)] \mathrm{bDGlcNAc}(1 \\
\rightarrow) \mathrm{N} 74\end{array}$ \\
\hline N122B & FA2 & $\begin{array}{l}\operatorname{bDGlcNAc}(1 \rightarrow 2) \mathrm{aDMan}(1 \rightarrow 6)[\mathrm{bDGlcNAc}(1 \rightarrow 2) \mathrm{aDMan}(1 \rightarrow 3)] \mathrm{bDMan}(1 \rightarrow 4) \mathrm{bDGlcNAc}(1 \rightarrow 4)[\operatorname{aLFuc}(1 \rightarrow \\
\text { 6)]bDGlcNAc}(1 \rightarrow) \mathrm{N} 122\end{array}$ \\
\hline N149B & FA3 & $\begin{array}{l}\operatorname{bDGlcNAc}(1 \rightarrow 6)[\mathrm{bDGlcNAc}(1 \rightarrow 2)] \mathrm{aDMan}(1 \rightarrow 6)[\mathrm{bDGlcNAc}(1 \rightarrow 2) \mathrm{aDMan}(1 \rightarrow 3)] \mathrm{bDMan}(1 \rightarrow 4) \mathrm{bDGlcNAc}(1 \rightarrow \\
\text { 4) }[\operatorname{aLFuc}(1 \rightarrow 6)] \mathrm{bDGlcNAc}(1 \rightarrow) \mathrm{N} 149\end{array}$ \\
\hline
\end{tabular}




\begin{tabular}{|c|c|c|}
\hline N165B & M5 & $\operatorname{aDMan}(1 \rightarrow 6)[\operatorname{aDMan}(1 \rightarrow 3)] \mathrm{aDMan}(1 \rightarrow 6)[\operatorname{aDMan}(1 \rightarrow 3)] \mathrm{bDMan}(1 \rightarrow 4) \mathrm{bDGlcNAc}(1 \rightarrow 4) \mathrm{bDGlcNAc}(1 \rightarrow) \mathrm{N} 165$ \\
\hline $\mathrm{N} 234 \mathrm{~B}$ & M9 & $\begin{array}{l}\operatorname{aDMan}(1 \rightarrow 2) \mathrm{aDMan}(1 \rightarrow 6)[\operatorname{aDMan}(1 \rightarrow 2) \mathrm{aDMan}(1 \rightarrow 3)] \mathrm{aDMan}(1 \rightarrow 6)[\operatorname{aDMan}(1 \rightarrow 2) \operatorname{aDMan}(1 \rightarrow 2) \mathrm{aDMan}(1 \rightarrow \\
\text { 3)]bDMan }(1 \rightarrow 4) \mathrm{bDGlcNAc}(1 \rightarrow 4) \mathrm{bDGlcNAc}(1 \rightarrow) \mathrm{N} 234\end{array}$ \\
\hline $\mathrm{N} 282 \mathrm{~B}$ & FA3G3S1 & $\begin{array}{l}\operatorname{aDNeu} 5 \operatorname{Ac}(2 \rightarrow 6) \mathrm{bDGal}(1 \rightarrow 4) \mathrm{bDGlcNAc}(1 \rightarrow 2) \mathrm{aDMan}(1 \rightarrow 3)[\mathrm{bDGal}(1 \rightarrow 4) \mathrm{bDGlcNAc}(1 \rightarrow 6)[\mathrm{bDGal}(1 \rightarrow \\
\text { 4)bDGlcNAc}(1 \rightarrow 2)] \mathrm{aDMan}(1 \rightarrow 6)] \mathrm{bDMan}(1 \rightarrow 4) \mathrm{bDGlcNAc}(1 \rightarrow 4)[\operatorname{aLFuc}(1 \rightarrow 6)] \mathrm{bDGlcNAc}(1 \rightarrow) \mathrm{N} 282\end{array}$ \\
\hline N331B & FA2 & $\begin{array}{l}\operatorname{bDGlcNAc}(1 \rightarrow 2) \mathrm{aDMan}(1 \rightarrow 6)[\mathrm{bDGlcNAc}(1 \rightarrow 2) \mathrm{aDMan}(1 \rightarrow 3)] \mathrm{bDMan}(1 \rightarrow 4) \mathrm{bDGlcNAc}(1 \rightarrow 4)[\mathrm{aLFuc}(1 \rightarrow \\
\text { 6)]bDGlcNAc}(1 \rightarrow) \mathrm{N} 331\end{array}$ \\
\hline N343B & FA1 & $\begin{array}{l}\operatorname{bDGlcNAc}(1 \rightarrow 2) \operatorname{aDMan}(1 \rightarrow 3)[\operatorname{aDMan}(1 \rightarrow 6)] \mathrm{bDMan}(1 \rightarrow 4) \mathrm{bDGlcNAc}(1 \rightarrow 4)[\operatorname{aLFuc}(1 \rightarrow 6)] \mathrm{bDGlcNAc}(1 \\
\rightarrow) \mathrm{N} 343\end{array}$ \\
\hline N603B & M5 & $\operatorname{aDMan}(1 \rightarrow 6)[\operatorname{aDMan}(1 \rightarrow 3)] \mathrm{aDMan}(1 \rightarrow 6)[\operatorname{aDMan}(1 \rightarrow 3)] \mathrm{bDMan}(1 \rightarrow 4) \mathrm{bDGlcNAc}(1 \rightarrow 4) \mathrm{bDGlcNAc}(1 \rightarrow) \mathrm{N} 603$ \\
\hline N616B & FA2 & $\begin{array}{l}\text { bDGlcNAc }(1 \rightarrow 2) \mathrm{aDMan}(1 \rightarrow 6)[\mathrm{bDGlcNAc}(1 \rightarrow 2) \mathrm{aDMan}(1 \rightarrow 3)] \mathrm{bDMan}(1 \rightarrow 4) \mathrm{bDGlcNAc}(1 \rightarrow 4)[\mathrm{aLFuc}(1 \rightarrow \\
\text { 6)]bDGlcNAc}(1 \rightarrow) \mathrm{N} 616\end{array}$ \\
\hline N657B & Hybrid G1 & $\begin{array}{l}\operatorname{bDGal}(1 \rightarrow 4) \mathrm{bDGlcNAc}(1 \rightarrow 2) \operatorname{aDMan}(1 \rightarrow 3)[\operatorname{aDMan}(1 \rightarrow 6)[\operatorname{aDMan}(1 \rightarrow 3)] \operatorname{aDMan}(1 \rightarrow 6)] \mathrm{bDMan}(1 \rightarrow \\
\text { 4)bDGlcNAc}(1 \rightarrow 4) \mathrm{bDGlcNAc}(1 \rightarrow) \mathrm{N} 657\end{array}$ \\
\hline N709B & M5 & $\operatorname{aDMan}(1 \rightarrow 6)[\operatorname{aDMan}(1 \rightarrow 3)] \mathrm{aDMan}(1 \rightarrow 6)[\operatorname{aDMan}(1 \rightarrow 3)] \mathrm{bDMan}(1 \rightarrow 4) \mathrm{bDGlcNAc}(1 \rightarrow 4) \mathrm{bDGlcNAc}(1 \rightarrow) \mathrm{N} 709$ \\
\hline N717B & M5 & $\operatorname{aDMan}(1 \rightarrow 6)[\operatorname{aDMan}(1 \rightarrow 3)] \mathrm{aDMan}(1 \rightarrow 6)[\operatorname{aDMan}(1 \rightarrow 3)] \mathrm{bDMan}(1 \rightarrow 4) \mathrm{bDGlcNAc}(1 \rightarrow 4) \mathrm{bDGlcNAc}(1 \rightarrow) \mathrm{N} 717$ \\
\hline N801B & M7 & $\begin{array}{l}\mathrm{aDMan}(1 \rightarrow 2) \mathrm{aDMan}(1 \rightarrow 2) \mathrm{aDMan}(1 \rightarrow 3)[\mathrm{aDMan}(1 \rightarrow 6)[\mathrm{aDMan}(1 \rightarrow 3)] \mathrm{aDMan}(1 \rightarrow 6)] \mathrm{bDMan}(1 \rightarrow 4) \mathrm{bDGlcNAc}(1 \\
\rightarrow 4) \mathrm{bDGlcNAc}(1 \rightarrow) \mathrm{N} 801\end{array}$ \\
\hline N1074B & M5 & $\operatorname{aDMan}(1 \rightarrow 6)[\operatorname{aDMan}(1 \rightarrow 3)] \mathrm{aDMan}(1 \rightarrow 6)[\operatorname{aDMan}(1 \rightarrow 3)] \mathrm{bDMan}(1 \rightarrow 4) \mathrm{bDGlcNAc}(1 \rightarrow 4) \mathrm{bDGlcNAc}(1 \rightarrow) \mathrm{N} 1074$ \\
\hline N1098B & A2 & $\begin{array}{l}\operatorname{bDGlcNAc}(1 \rightarrow 2) \mathrm{aDMan}(1 \rightarrow 6)[\mathrm{bDGlcNAc}(1 \rightarrow 2) \mathrm{aDMan}(1 \rightarrow 3)] \mathrm{bDMan}(1 \rightarrow 4) \mathrm{bDGlcNAc}(1 \rightarrow 4) \mathrm{bDGlcNAc}(1 \\
\rightarrow) \mathrm{N} 1098\end{array}$ \\
\hline N61C & M5 & $\operatorname{aDMan}(1 \rightarrow 6)[\operatorname{aDMan}(1 \rightarrow 3)] \mathrm{aDMan}(1 \rightarrow 6)[\operatorname{aDMan}(1 \rightarrow 3)] \mathrm{bDMan}(1 \rightarrow 4) \mathrm{bDGlcNAc}(1 \rightarrow 4) \mathrm{bDGlcNAc}(1 \rightarrow) \mathrm{N} 61$ \\
\hline $\mathrm{N} 74 \mathrm{C}$ & A2 & $\begin{array}{l}\text { bDGlcNAc }(1 \rightarrow 2) \mathrm{aDMan}(1 \rightarrow 6)[\mathrm{bDGlcNAc}(1 \rightarrow 2) \mathrm{aDMan}(1 \rightarrow 3)] \mathrm{bDMan}(1 \rightarrow 4) \mathrm{bDGlcNAc}(1 \rightarrow 4) \mathrm{bDGlcNAc}(1 \\
\rightarrow) \mathrm{N} 74\end{array}$ \\
\hline $\mathrm{N} 122 \mathrm{C}$ & M5 & $\operatorname{aDMan}(1 \rightarrow 6)[\operatorname{aDMan}(1 \rightarrow 3)] \mathrm{aDMan}(1 \rightarrow 6)[\operatorname{aDMan}(1 \rightarrow 3)] \mathrm{bDMan}(1 \rightarrow 4) \mathrm{bDGlcNAc}(1 \rightarrow 4) \mathrm{bDGlcNAc}(1 \rightarrow) \mathrm{N} 122$ \\
\hline $\mathrm{N} 149 \mathrm{C}$ & FA2 & $\begin{array}{l}\operatorname{bDGlcNAc}(1 \rightarrow 2) \mathrm{aDMan}(1 \rightarrow 6)[\mathrm{bDGlcNAc}(1 \rightarrow 2) \mathrm{aDMan}(1 \rightarrow 3)] \mathrm{bDMan}(1 \rightarrow 4) \mathrm{bDGlcNAc}(1 \rightarrow 4)[\mathrm{aLFuc}(1 \rightarrow \\
\text { 6)]bDGlcNAc}(1 \rightarrow) \mathrm{N} 149\end{array}$ \\
\hline $\mathrm{N} 165 \mathrm{C}$ & FA2G2S1 & $\begin{array}{l}\mathrm{aDNeu} 5 \mathrm{Ac}(2 \rightarrow 6) \mathrm{bDGal}(1 \rightarrow 4) \mathrm{bDGlcNAc}(1 \rightarrow 2) \mathrm{aDMan}(1 \rightarrow 6)[\mathrm{bDGal}(1 \rightarrow 4) \mathrm{bDGlcNAc}(1 \rightarrow 2) \mathrm{aDMan}(1 \rightarrow \\
\text { 3)]bDMan }(1 \rightarrow 4) \mathrm{bDGlcNAc}(1 \rightarrow 4)[\operatorname{aLFuc}(1 \rightarrow 6)] \mathrm{bDGlcNAc}(1 \rightarrow) \mathrm{N} 165\end{array}$ \\
\hline $\mathrm{N} 234 \mathrm{C}$ & M9 & $\begin{array}{l}\operatorname{aDMan}(1 \rightarrow 2) \mathrm{aDMan}(1 \rightarrow 6)[\operatorname{aDMan}(1 \rightarrow 2) \mathrm{aDMan}(1 \rightarrow 3)] \mathrm{aDMan}(1 \rightarrow 6)[\operatorname{aDMan}(1 \rightarrow 2) \mathrm{aDMan}(1 \rightarrow 2) \mathrm{aDMan}(1 \rightarrow \\
\text { 3)]bDMan }(1 \rightarrow 4) \mathrm{bDGlcNAc}(1 \rightarrow 4) \mathrm{bDGlcNAc}(1 \rightarrow) \mathrm{N} 234\end{array}$ \\
\hline $\mathrm{N} 282 \mathrm{C}$ & $\mathrm{A} 2$ & $\begin{array}{l}\operatorname{bDGlcNAc}(1 \rightarrow 2) \mathrm{aDMan}(1 \rightarrow 6)[\mathrm{bDGlcNAc}(1 \rightarrow 2) \mathrm{aDMan}(1 \rightarrow 3)] \mathrm{bDMan}(1 \rightarrow 4) \mathrm{bDGlcNAc}(1 \rightarrow 4) \mathrm{bDGlcNAc}(1 \\
\rightarrow) \mathrm{N} 282\end{array}$ \\
\hline N331C & FA3G3S1 & $\begin{array}{l}\operatorname{aDNeu} 5 \operatorname{Ac}(2 \rightarrow 6) \mathrm{bDGal}(1 \rightarrow 4) \mathrm{bDGlcNAc}(1 \rightarrow 2) \mathrm{aDMan}(1 \rightarrow 3)[\mathrm{bDGal}(1 \rightarrow 4) \operatorname{bDGlcNAc}(1 \rightarrow 6)[\mathrm{bDGal}(1 \rightarrow \\
\text { 4)bDGlcNAc}(1 \rightarrow 2)] \mathrm{aDMan}(1 \rightarrow 6)] \mathrm{bDMan}(1 \rightarrow 4) \mathrm{bDGlcNAc}(1 \rightarrow 4)[\operatorname{aLFuc}(1 \rightarrow 6)] \mathrm{bDGlcNAc}(1 \rightarrow) \mathrm{N} 331\end{array}$ \\
\hline N343C & FA2 & $\begin{array}{l}\text { bDGlcNAc }(1 \rightarrow 2) \mathrm{aDMan}(1 \rightarrow 6)[\mathrm{bDGlcNAc}(1 \rightarrow 2) \mathrm{aDMan}(1 \rightarrow 3)] \mathrm{bDMan}(1 \rightarrow 4) \mathrm{bDGlcNAc}(1 \rightarrow 4)[\operatorname{aLFuc}(1 \rightarrow \\
\text { 6)]bDGlcNAc}(1 \rightarrow) \mathrm{N} 343\end{array}$ \\
\hline N603C & M5 & $\operatorname{aDMan}(1 \rightarrow 6)[\operatorname{aDMan}(1 \rightarrow 3)] \mathrm{aDMan}(1 \rightarrow 6)[\operatorname{aDMan}(1 \rightarrow 3)] \mathrm{bDMan}(1 \rightarrow 4) \mathrm{bDGlcNAc}(1 \rightarrow 4) \mathrm{bDGlcNAc}(1 \rightarrow) \mathrm{N} 603$ \\
\hline N616C & FA2 & $\begin{array}{l}\text { bDGlcNAc }(1 \rightarrow 2) \mathrm{aDMan}(1 \rightarrow 6)[\mathrm{bDGlcNAc}(1 \rightarrow 2) \mathrm{aDMan}(1 \rightarrow 3)] \mathrm{bDMan}(1 \rightarrow 4) \mathrm{bDGlcNAc}(1 \rightarrow 4)[\mathrm{aLFuc}(1 \rightarrow \\
\text { 6)]bDGlcNAc}(1 \rightarrow) \mathrm{N} 616\end{array}$ \\
\hline $\mathrm{N} 657 \mathrm{C}$ & Hybrid G1 & $\begin{array}{l}\operatorname{bDGal}(1 \rightarrow 4) \mathrm{bDGlcNAc}(1 \rightarrow 2) \mathrm{aDMan}(1 \rightarrow 3)[\operatorname{aDMan}(1 \rightarrow 6)[\operatorname{aDMan}(1 \rightarrow 3)] \mathrm{aDMan}(1 \rightarrow 6)] \mathrm{bDMan}(1 \rightarrow \\
\text { 4)bDGlcNAc}(1 \rightarrow 4) \mathrm{bDGlcNAc}(1 \rightarrow) \mathrm{N} 657\end{array}$ \\
\hline N709C & M5 & $\operatorname{aDMan}(1 \rightarrow 6)[\operatorname{aDMan}(1 \rightarrow 3)] \mathrm{aDMan}(1 \rightarrow 6)[\operatorname{aDMan}(1 \rightarrow 3)] \mathrm{bDMan}(1 \rightarrow 4) \mathrm{bDGlcNAc}(1 \rightarrow 4) \mathrm{bDGlcNAc}(1 \rightarrow) \mathrm{N} 709$ \\
\hline
\end{tabular}




\begin{tabular}{|l|l|l|}
\hline N717C & M6 & $\begin{array}{l}\operatorname{aDMan}(1 \rightarrow 6)[\operatorname{aDMan}(1 \rightarrow 3)] \mathrm{aDMan}(1 \rightarrow 6)[\operatorname{aDMan}(1 \rightarrow 2) \mathrm{aDMan}(1 \rightarrow 3)] \mathrm{bDMan}(1 \rightarrow 4) \mathrm{bDGlcNAc}(1 \rightarrow \\
\text { 4)bDGlcNAc}(1 \rightarrow) \mathrm{N} 717\end{array}$ \\
\hline $\mathrm{N} 801 \mathrm{C}$ & M5 & $\mathrm{aDMan}(1 \rightarrow 6)[\operatorname{aDMan}(1 \rightarrow 3)] \mathrm{aDMan}(1 \rightarrow 6)[\operatorname{aDMan}(1 \rightarrow 3)] \mathrm{bDMan}(1 \rightarrow 4) \mathrm{bDGlcNAc}(1 \rightarrow 4) \mathrm{bDGlcNAc}(1 \rightarrow) \mathrm{N} 801$ \\
\hline $\mathrm{N} 1074 \mathrm{C}$ & M5 & $\mathrm{aDMan}(1 \rightarrow 6)[\operatorname{aDMan}(1 \rightarrow 3)] \mathrm{aDMan}(1 \rightarrow 6)[\operatorname{aDMan}(1 \rightarrow 3)] \mathrm{bDMan}(1 \rightarrow 4) \mathrm{bDGlcNAc}(1 \rightarrow 4) \mathrm{bDGlcNAc}(1 \rightarrow) \mathrm{N} 1074$ \\
\hline $\mathrm{N} 1098 \mathrm{C}$ & $\begin{array}{l}\text { Hybrid } \\
\text { G1S1 }\end{array}$ & $\begin{array}{l}\mathrm{aDNeu} 5 \mathrm{Ac}(2 \rightarrow 6) \mathrm{bDGal}(1 \rightarrow 4) \mathrm{bDGlcNAc}(1 \rightarrow 2) \mathrm{aDMan}(1 \rightarrow 3)[\operatorname{aDMan}(1 \rightarrow 6)[\mathrm{aDMan}(1 \rightarrow 3)] \mathrm{aDMan}(1 \rightarrow \\
\text { 6)]bDMan}(1 \rightarrow 4) \mathrm{bDGlcNAc}(1 \rightarrow 4) \mathrm{bDGlcNAc}(1 \rightarrow) \mathrm{N} 1098\end{array}$ \\
\hline
\end{tabular}

Table S6. The glycosylation of CoV-S according to experimental data ${ }^{43}$.

\begin{tabular}{|c|c|c|}
\hline Residues & Type & Glycan \\
\hline N29A & FA3 & $\begin{array}{l}\operatorname{bDGlcNAc}(1 \rightarrow 6)[\mathrm{bDGlcNAc}(1 \rightarrow 2)] \mathrm{aDMan}(1 \rightarrow 6)[\mathrm{bDGlcNAc}(1 \rightarrow 2) \operatorname{aDMan}(1 \rightarrow 3)] \mathrm{bDMan}(1 \rightarrow \\
\text { 4)bDGlcNAc}(1 \rightarrow 4)[\operatorname{aLFuc}(1 \rightarrow 6)] \mathrm{bDGlcNAc}(1 \rightarrow) \mathrm{N} 29\end{array}$ \\
\hline N65A & M6 & $\begin{array}{l}\operatorname{aDMan}(1 \rightarrow 6)[\operatorname{aDMan}(1 \rightarrow 3)] \mathrm{aDMan}(1 \rightarrow 6)[\mathrm{aDMan}(1 \rightarrow 2) \mathrm{aDMan}(1 \rightarrow 3)] \mathrm{bDMan}(1 \rightarrow 4) \mathrm{bDGlcNAc}(1 \rightarrow \\
\text { 4)bDGlcNAc}(1 \rightarrow) \mathrm{N} 65\end{array}$ \\
\hline N73A & FA3G3S1 & $\begin{array}{l}\mathrm{aDNeu} 5 \operatorname{Ac}(2 \rightarrow 6) \mathrm{bDGal}(1 \rightarrow 4) \mathrm{bDGlcNAc}(1 \rightarrow 2) \mathrm{aDMan}(1 \rightarrow 3)[\mathrm{bDGal}(1 \rightarrow 4) \mathrm{bDGlcNAc}(1 \rightarrow 6)[\mathrm{bDGal}(1 \rightarrow \\
\text { 4)bDGlcNAc}(1 \rightarrow 2)] \mathrm{aDMan}(1 \rightarrow 6)] \mathrm{bDMan}(1 \rightarrow 4) \mathrm{bDGlcNAc}(1 \rightarrow 4)[\operatorname{aLFuc}(1 \rightarrow 6)] \mathrm{bDGlcNAc}(1 \rightarrow) \mathrm{N} 73\end{array}$ \\
\hline N109A & FA3 & $\begin{array}{l}\operatorname{bDGlcNAc}(1 \rightarrow 6)[\mathrm{bDGlcNAc}(1 \rightarrow 2)] \mathrm{aDMan}(1 \rightarrow 6)[\mathrm{bDGlcNAc}(1 \rightarrow 2) \operatorname{aDMan}(1 \rightarrow 3)] \mathrm{bDMan}(1 \rightarrow \\
\text { 4)bDGlcNAc}(1 \rightarrow 4)[\operatorname{aLFuc}(1 \rightarrow 6)] \mathrm{bDGlcNAc}(1 \rightarrow) \mathrm{N} 109\end{array}$ \\
\hline N118A & FA2 & $\begin{array}{l}\text { bDGlcNAc }(1 \rightarrow 2) \operatorname{aDMan}(1 \rightarrow 6)[\mathrm{bDGlcNAc}(1 \rightarrow 2) \operatorname{aDMan}(1 \rightarrow 3)] \mathrm{bDMan}(1 \rightarrow 4) \mathrm{bDGlcNAc}(1 \rightarrow 4)[\operatorname{aLFuc}(1 \rightarrow \\
\text { 6)]bDGlcNAc}(1 \rightarrow) \operatorname{N} 118\end{array}$ \\
\hline N158A & M7 & $\begin{array}{l}\operatorname{aDMan}(1 \rightarrow 2) \mathrm{aDMan}(1 \rightarrow 2) \mathrm{aDMan}(1 \rightarrow 3)[\operatorname{aDMan}(1 \rightarrow 6)[\mathrm{aDMan}(1 \rightarrow 3)] \mathrm{aDMan}(1 \rightarrow 6)] \mathrm{bDMan}(1 \rightarrow \\
\text { 4)bDGlcNAc}(1 \rightarrow 4) \mathrm{bDGlcNAc}(1 \rightarrow) \mathrm{N} 158\end{array}$ \\
\hline $\mathrm{N} 227 \mathrm{~A}$ & M9 & $\begin{array}{l}\operatorname{aDMan}(1 \rightarrow 2) \mathrm{aDMan}(1 \rightarrow 6)[\operatorname{aDMan}(1 \rightarrow 2) \mathrm{aDMan}(1 \rightarrow 3)] \mathrm{aDMan}(1 \rightarrow 6)[\mathrm{aDMan}(1 \rightarrow 2) \mathrm{aDMan}(1 \rightarrow 2) \mathrm{aDMan}(1 \rightarrow \\
\text { 3)]bDMan }(1 \rightarrow 4) \mathrm{bDGlcNAc}(1 \rightarrow 4) \mathrm{bDGlcNAc}(1 \rightarrow) \mathrm{N} 227\end{array}$ \\
\hline N269A & FA3G3S1 & $\begin{array}{l}\operatorname{aDNeu} 5 \operatorname{Ac}(2 \rightarrow 6) \mathrm{bDGal}(1 \rightarrow 4) \mathrm{bDGlcNAc}(1 \rightarrow 2) \operatorname{aDMan}(1 \rightarrow 3)[\mathrm{bDGal}(1 \rightarrow 4) \mathrm{bDGlcNAc}(1 \rightarrow 6)[\mathrm{bDGal}(1 \rightarrow \\
\text { 4)bDGlcNAc}(1 \rightarrow 2)] \mathrm{aDMan}(1 \rightarrow 6)] \mathrm{bDMan}(1 \rightarrow 4) \mathrm{bDGlcNAc}(1 \rightarrow 4)[\operatorname{aLFuc}(1 \rightarrow 6)] \mathrm{bDGlcNAc}(1 \rightarrow) \mathrm{N} 269\end{array}$ \\
\hline N318A & FA2 & $\begin{array}{l}\text { bDGlcNAc}(1 \rightarrow 2) \operatorname{aDMan}(1 \rightarrow 6)[\operatorname{bDGlcNAc}(1 \rightarrow 2) \operatorname{aDMan}(1 \rightarrow 3)] \mathrm{bDMan}(1 \rightarrow 4) \mathrm{bDGlcNAc}(1 \rightarrow 4)[\operatorname{aLFuc}(1 \rightarrow \\
\text { 6)]bDGlcNAc}(1 \rightarrow) \mathrm{N} 318\end{array}$ \\
\hline N330A & FA2 & $\begin{array}{l}\text { bDGlcNAc }(1 \rightarrow 2) \operatorname{aDMan}(1 \rightarrow 6)[\mathrm{bDGlcNAc}(1 \rightarrow 2) \operatorname{aDMan}(1 \rightarrow 3)] \mathrm{bDMan}(1 \rightarrow 4) \mathrm{bDGlcNAc}(1 \rightarrow 4)[\operatorname{aLFuc}(1 \rightarrow \\
\text { 6)]bDGlcNAc}(1 \rightarrow) \mathrm{N} 330\end{array}$ \\
\hline N357A & FA2 & $\begin{array}{l}\text { bDGlcNAc}(1 \rightarrow 2) \operatorname{aDMan}(1 \rightarrow 6)[\operatorname{bDGlcNAc}(1 \rightarrow 2) \operatorname{aDMan}(1 \rightarrow 3)] \mathrm{bDMan}(1 \rightarrow 4) \mathrm{bDGlcNAc}(1 \rightarrow 4)[\operatorname{aLFuc}(1 \rightarrow \\
\text { 6)]bDGlcNAc}(1 \rightarrow) \mathrm{N} 357\end{array}$ \\
\hline N589A & M5 & $\begin{array}{l}\operatorname{aDMan}(1 \rightarrow 6)[\operatorname{aDMan}(1 \rightarrow 3)] \operatorname{aDMan}(1 \rightarrow 6)[\operatorname{aDMan}(1 \rightarrow 3)] \mathrm{bDMan}(1 \rightarrow 4) \mathrm{bDGlcNAc}(1 \rightarrow 4) \mathrm{bDGlcNAc}(1 \\
\rightarrow) \mathrm{N} 589\end{array}$ \\
\hline N602A & $\mathrm{A} 2$ & $\begin{array}{l}\text { bDGlcNAc }(1 \rightarrow 2) \mathrm{aDMan}(1 \rightarrow 6)[\mathrm{bDGlcNAc}(1 \rightarrow 2) \mathrm{aDMan}(1 \rightarrow 3)] \mathrm{bDMan}(1 \rightarrow 4) \mathrm{bDGlcNAc}(1 \rightarrow 4) \mathrm{bDGlcNAc}(1 \\
\rightarrow) \mathrm{N} 602\end{array}$ \\
\hline N691A & FA2 & $\begin{array}{l}\text { bDGlcNAc }(1 \rightarrow 2) \operatorname{aDMan}(1 \rightarrow 6)[\operatorname{bDGlcNAc}(1 \rightarrow 2) \operatorname{aDMan}(1 \rightarrow 3)] \mathrm{bDMan}(1 \rightarrow 4) \mathrm{bDGlcNAc}(1 \rightarrow 4)[\operatorname{aLFuc}(1 \rightarrow \\
\text { 6)]bDGlcNAc}(1 \rightarrow) \operatorname{N691}\end{array}$ \\
\hline N699A & FA3G3S1 & $\begin{array}{l}\operatorname{aDNeu} 5 \operatorname{Ac}(2 \rightarrow 6) \mathrm{bDGa}(1 \rightarrow 4) \mathrm{bDGlcNAc}(1 \rightarrow 2) \operatorname{aDMan}(1 \rightarrow 3)[\mathrm{bDGal}(1 \rightarrow 4) \mathrm{bDGlcNAc}(1 \rightarrow 6)[\mathrm{bDGal}(1 \rightarrow \\
\text { 4)bDGlcNAc}(1 \rightarrow 2)] \mathrm{aDMan}(1 \rightarrow 6)] \mathrm{bDMan}(1 \rightarrow 4) \mathrm{bDGlcNAc}(1 \rightarrow 4)[\operatorname{aLFuc}(1 \rightarrow 6)] \mathrm{bDGlcNAc}(1 \rightarrow) \operatorname{N} 699\end{array}$ \\
\hline N783A & M7 & $\begin{array}{l}\operatorname{aDMan}(1 \rightarrow 2) \mathrm{aDMan}(1 \rightarrow 2) \mathrm{aDMan}(1 \rightarrow 3)[\operatorname{aDMan}(1 \rightarrow 6)[\mathrm{aDMan}(1 \rightarrow 3)] \mathrm{aDMan}(1 \rightarrow 6)] \mathrm{bDMan}(1 \rightarrow \\
\text { 4)bDGlcNAc}(1 \rightarrow 4) \mathrm{bDGlcNAc}(1 \rightarrow) \mathrm{N} 783\end{array}$ \\
\hline N1056A & FA3 & $\begin{array}{l}\operatorname{bDGlcNAc}(1 \rightarrow 6)[\operatorname{bDGlcNAc}(1 \rightarrow 2)] \mathrm{aDMan}(1 \rightarrow 6)[\mathrm{bDGlcNAc}(1 \rightarrow 2) \operatorname{aDMan}(1 \rightarrow 3)] \mathrm{bDMan}(1 \rightarrow \\
\text { 4)bDGlcNAc}(1 \rightarrow 4)[\operatorname{aLFuc}(1 \rightarrow 6)] \mathrm{bDGlcNAc}(1 \rightarrow) \mathrm{N} 1056\end{array}$ \\
\hline
\end{tabular}




\begin{tabular}{|c|c|c|}
\hline N1080A & Hydrid G1 & $\begin{array}{l}\operatorname{bDGal}(1 \rightarrow 4) \mathrm{bDGlcNAc}(1 \rightarrow 2) \mathrm{aDMan}(1 \rightarrow 3)[\operatorname{aDMan}(1 \rightarrow 6)[\operatorname{aDMan}(1 \rightarrow 3)] \mathrm{aDMan}(1 \rightarrow 6)] \mathrm{bDMan}(1 \rightarrow \\
\text { 4)bDGlcNAc}(1 \rightarrow 4) \mathrm{bDGlcNAc}(1 \rightarrow) \mathrm{N} 1080\end{array}$ \\
\hline $\mathrm{N} 29 \mathrm{~B}$ & FA3 & $\begin{array}{l}\operatorname{bDGlcNAc}(1 \rightarrow 6)[\mathrm{bDGlcNAc}(1 \rightarrow 2)] \mathrm{aDMan}(1 \rightarrow 6) \quad[\mathrm{bDGlcNAc}(1 \rightarrow 2) \mathrm{aDMan}(1 \rightarrow 3)] \mathrm{bDMan}(1 \rightarrow \\
\text { 4)bDGlcNAc}(1 \rightarrow 4)[\operatorname{aLFuc}(1 \rightarrow 6)] \mathrm{bDGlcNAc}(1 \rightarrow) \mathrm{N} 29\end{array}$ \\
\hline N65B & M6 & $\begin{array}{l}\operatorname{aDMan}(1 \rightarrow 6)[\operatorname{aDMan}(1 \rightarrow 3)] \mathrm{aDMan}(1 \rightarrow 6)[\mathrm{aDMan}(1 \rightarrow 2) \mathrm{aDMan}(1 \rightarrow 3)] \mathrm{bDMan}(1 \rightarrow 4) \mathrm{bDGlcNAc}(1 \rightarrow \\
\text { 4)bDGlcNAc}(1 \rightarrow) \mathrm{N} 65\end{array}$ \\
\hline N73B & FA2G2S1 & $\begin{array}{l}\text { aDNeu5Ac }(2 \rightarrow 6) \mathrm{bDGal}(1 \rightarrow 4) \operatorname{bDGlcNAc}(1 \rightarrow 2) \operatorname{aDMan}(1 \rightarrow 3)[\mathrm{bDGal}(1 \rightarrow 4) \operatorname{bDGlcNAc}(1 \rightarrow 2) \operatorname{aDMan}(1 \rightarrow \\
\text { 6)]bDMan }(1 \rightarrow 4) \mathrm{bDGlcNAc}(1 \rightarrow 4)[\operatorname{aLFuc}(1 \rightarrow 6)] \mathrm{bDGlcNAc}(1 \rightarrow) \mathrm{N} 73\end{array}$ \\
\hline N109B & FA3 & $\begin{array}{l}\operatorname{bDGlcNAc}(1 \rightarrow 6)[\operatorname{bDGlcNAc}(1 \rightarrow 2)] \operatorname{aDMan}(1 \rightarrow 6) \quad[\operatorname{bDGlcNAc}(1 \rightarrow 2) \operatorname{aDMan}(1 \rightarrow 3)] \mathrm{bDMan}(1 \rightarrow \\
\text { 4)bDGlcNAc}(1 \rightarrow 4)[\operatorname{aLFuc}(1 \rightarrow 6)] \mathrm{bDGlcNAc}(1 \rightarrow) \mathrm{N} 109\end{array}$ \\
\hline N118B & FA3 & $\begin{array}{l}\operatorname{bDGlcNAc}(1 \rightarrow 6)[\mathrm{bDGlcNAc}(1 \rightarrow 2)] \mathrm{aDMan}(1 \rightarrow 6) \quad[\mathrm{bDGlcNAc}(1 \rightarrow 2) \mathrm{aDMan}(1 \rightarrow 3)] \mathrm{bDMan}(1 \rightarrow \\
\text { 4)bDGlcNAc}(1 \rightarrow 4)[\operatorname{aLFuc}(1 \rightarrow 6)] \mathrm{bDGlcNAc}(1 \rightarrow) \mathrm{N} 118\end{array}$ \\
\hline N158B & M6 & $\begin{array}{l}\operatorname{aDMan}(1 \rightarrow 6)[\operatorname{aDMan}(1 \rightarrow 3)] \mathrm{aDMan}(1 \rightarrow 6)[\operatorname{aDMan}(1 \rightarrow 2) \mathrm{aDMan}(1 \rightarrow 3)] \mathrm{bDMan}(1 \rightarrow 4) \mathrm{bDGlcNAc}(1 \rightarrow \\
\text { 4)bDGlcNAc}(1 \rightarrow) \mathrm{N} 158\end{array}$ \\
\hline N227B & M9 & $\begin{array}{l}\operatorname{aDMan}(1 \rightarrow 2) \mathrm{aDMan}(1 \rightarrow 6)[\mathrm{aDMan}(1 \rightarrow 2) \mathrm{aDMan}(1 \rightarrow 3)] \mathrm{aDMan}(1 \rightarrow 6)[\mathrm{aDMan}(1 \rightarrow 2) \mathrm{aDMan}(1 \rightarrow 2) \mathrm{aDMan}(1 \rightarrow \\
\text { 3)]bDMan}(1 \rightarrow 4) \mathrm{bDGlcNAc}(1 \rightarrow 4) \mathrm{bDGlcNAc}(1 \rightarrow) \mathrm{N} 227\end{array}$ \\
\hline N269B & FA3G3S1 & $\begin{array}{l}\mathrm{aDNeu} 5 \operatorname{Ac}(2 \rightarrow 6) \mathrm{bDGal}(1 \rightarrow 4) \mathrm{bDGlcNAc}(1 \rightarrow 2) \mathrm{aDMan}(1 \rightarrow 3)[\mathrm{bDGal}(1 \rightarrow 4) \mathrm{bDGlcNAc}(1 \rightarrow 6)[\mathrm{bDGal}(1 \rightarrow \\
\text { 4)bDGlcNAc}(1 \rightarrow 2)] \mathrm{aDMan}(1 \rightarrow 6)] \mathrm{bDMan}(1 \rightarrow 4) \mathrm{bDGlcNAc}(1 \rightarrow 4)[\operatorname{aLFuc}(1 \rightarrow 6)] \mathrm{bDGlcNAc}(1 \rightarrow) \mathrm{N} 269\end{array}$ \\
\hline N318B & FA3 & $\begin{array}{l}\operatorname{bDGlcNAc}(1 \rightarrow 6)[\operatorname{bDGlcNAc}(1 \rightarrow 2)] \operatorname{aDMan}(1 \rightarrow 6) \quad[\operatorname{bDGlcNAc}(1 \rightarrow 2) \mathrm{aDMan}(1 \rightarrow 3)] \mathrm{bDMan}(1 \rightarrow \\
\text { 4)bDGlcNAc}(1 \rightarrow 4)[\operatorname{aLFuc}(1 \rightarrow 6)] \mathrm{bDGlcNAc}(1 \rightarrow) \mathrm{N} 318\end{array}$ \\
\hline N330B & FA2 & $\begin{array}{l}\operatorname{bDGlcNAc}(1 \rightarrow 2) \mathrm{aDMan}(1 \rightarrow 6)[\mathrm{bDGlcNAc}(1 \rightarrow 2) \mathrm{aDMan}(1 \rightarrow 3)] \mathrm{bDMan}(1 \rightarrow 4) \operatorname{bDGlcNAc}(1 \rightarrow 4)[\operatorname{aLFuc}(1 \rightarrow \\
\text { 6)]bDGlcNAc}(1 \rightarrow) \mathrm{N} 330\end{array}$ \\
\hline N357B & FA2 & $\begin{array}{l}\text { bDGlcNAc }(1 \rightarrow 2) \mathrm{aDMan}(1 \rightarrow 6)[\mathrm{bDGlcNAc}(1 \rightarrow 2) \mathrm{aDMan}(1 \rightarrow 3)] \mathrm{bDMan}(1 \rightarrow 4) \mathrm{bDGlcNAc}(1 \rightarrow 4)[\operatorname{aLFuc}(1 \rightarrow \\
\text { 6)]bDGlcNAc}(1 \rightarrow) \mathrm{N} 357\end{array}$ \\
\hline N589B & M5 & $\begin{array}{l}\operatorname{aDMan}(1 \rightarrow 6)[\operatorname{aDMan}(1 \rightarrow 3)] \mathrm{aDMan}(1 \rightarrow 6)[\operatorname{aDMan}(1 \rightarrow 3)] \mathrm{bDMan}(1 \rightarrow 4) \mathrm{bDGlcNAc}(1 \rightarrow 4) \mathrm{bDGlcNAc}(1 \\
\rightarrow) \mathrm{N} 589\end{array}$ \\
\hline N602B & A2 & $\begin{array}{l}\operatorname{bDGlcNAc}(1 \rightarrow 2) \mathrm{aDMan}(1 \rightarrow 6)[\mathrm{bDGlcNAc}(1 \rightarrow 2) \mathrm{aDMan}(1 \rightarrow 3)] \mathrm{bDMan}(1 \rightarrow 4) \mathrm{bDGlcNAc}(1 \rightarrow 4) \mathrm{bDGlcNAc}(1 \\
\rightarrow) \mathrm{N} 602\end{array}$ \\
\hline N691B & FA3 & $\begin{array}{l}\operatorname{bDGlcNAc}(1 \rightarrow 6)[\mathrm{bDGlcNAc}(1 \rightarrow 2)] \mathrm{aDMan}(1 \rightarrow 6) \quad[\mathrm{bDGlcNAc}(1 \rightarrow 2) \mathrm{aDMan}(1 \rightarrow 3)] \mathrm{bDMan}(1 \rightarrow \\
\text { 4)bDGlcNAc}(1 \rightarrow 4)[\operatorname{aLFuc}(1 \rightarrow 6)] \mathrm{bDGlcNAc}(1 \rightarrow) \mathrm{N} 691\end{array}$ \\
\hline N699B & A 3 & $\begin{array}{l}\operatorname{bDGlcNAc}(1 \rightarrow 6)[\operatorname{bDGlcNAc}(1 \rightarrow 2)] \operatorname{aDMan}(1 \rightarrow 6)[\operatorname{bDGlcNAc}(1 \rightarrow 2) \operatorname{aDMan}(1 \rightarrow 3)] \mathrm{bDMan}(1 \rightarrow \\
\text { 4)bDGlcNAc}(1 \rightarrow 4) \operatorname{bDGlcNAc}(1 \rightarrow) \mathrm{N} 699\end{array}$ \\
\hline N783B & M5 & $\begin{array}{l}\operatorname{aDMan}(1 \rightarrow 6)[\operatorname{aDMan}(1 \rightarrow 3)] \mathrm{aDMan}(1 \rightarrow 6)[\operatorname{aDMan}(1 \rightarrow 3)] \mathrm{bDMan}(1 \rightarrow 4) \mathrm{bDGlcNAc}(1 \rightarrow 4) \mathrm{bDGlcNAc}(1 \\
\rightarrow) \mathrm{N} 783\end{array}$ \\
\hline N1056B & FA3 & $\begin{array}{l}\operatorname{bDGlcNAc}(1 \rightarrow 6)[\mathrm{bDGlcNAc}(1 \rightarrow 2)] \operatorname{aDMan}(1 \rightarrow 6) \quad[\mathrm{bDGlcNAc}(1 \rightarrow 2) \mathrm{aDMan}(1 \rightarrow 3)] \mathrm{bDMan}(1 \rightarrow \\
\text { 4)bDGlcNAc}(1 \rightarrow 4)[\operatorname{aLFuc}(1 \rightarrow 6)] \mathrm{bDGlcNAc}(1 \rightarrow) \mathrm{N} 1056\end{array}$ \\
\hline N1080B & Hydrid G1 & $\begin{array}{l}\operatorname{bDGal}(1 \rightarrow 4) \mathrm{bDGlcNAc}(1 \rightarrow 2) \mathrm{aDMan}(1 \rightarrow 3)[\operatorname{aDMan}(1 \rightarrow 6)[\operatorname{aDMan}(1 \rightarrow 3)] \mathrm{aDMan}(1 \rightarrow 6)] \mathrm{bDMan}(1 \rightarrow \\
\text { 4)bDGlcNAc}(1 \rightarrow 4) \mathrm{bDGlcNAc}(1 \rightarrow) \mathrm{N} 1080\end{array}$ \\
\hline $\mathrm{N} 29 \mathrm{C}$ & FA3G3S1 & $\begin{array}{l}\operatorname{aDNeu} 5 \operatorname{Ac}(2 \rightarrow 6) \mathrm{bDGal}(1 \rightarrow 4) \mathrm{bDGlcNAc}(1 \rightarrow 2) \mathrm{aDMan}(1 \rightarrow 3)[\mathrm{bDGal}(1 \rightarrow 4) \mathrm{bDGlcNAc}(1 \rightarrow 6)[\mathrm{bDGal}(1 \rightarrow \\
\text { 4)bDGlcNAc}(1 \rightarrow 2)] \mathrm{aDMan}(1 \rightarrow 6)] \mathrm{bDMan}(1 \rightarrow 4) \mathrm{bDGlcNAc}(1 \rightarrow 4)[\operatorname{aLFuc}(1 \rightarrow 6)] \mathrm{bDGlcNAc}(1 \rightarrow) \mathrm{N} 29\end{array}$ \\
\hline N65C & M6 & $\begin{array}{l}\operatorname{aDMan}(1 \rightarrow 6)[\operatorname{aDMan}(1 \rightarrow 3)] \mathrm{aDMan}(1 \rightarrow 6)[\operatorname{aDMan}(1 \rightarrow 2) \mathrm{aDMan}(1 \rightarrow 3)] \mathrm{bDMan}(1 \rightarrow 4) \mathrm{bDGlcNAc}(1 \rightarrow \\
\text { 4)bDGlcNAc}(1 \rightarrow) \mathrm{N} 65\end{array}$ \\
\hline $\mathrm{N} 73 \mathrm{C}$ & FA3G3S1 & $\mathrm{aDNeu} 5 \mathrm{Ac}(2 \rightarrow 6) \mathrm{bDGal}(1 \rightarrow 4) \mathrm{bDGlcNAc}(1 \rightarrow 2) \mathrm{aDMan}(1 \rightarrow 3)[\mathrm{bDGal}(1 \rightarrow 4) \mathrm{bDGlcNAc}(1 \rightarrow 6)[\mathrm{bDGal}(1 \rightarrow$ \\
\hline
\end{tabular}




\begin{tabular}{|c|c|c|}
\hline & & 4)bDGlcNAc $(1 \rightarrow 2)] \mathrm{aDMan}(1 \rightarrow 6)] \mathrm{bDMan}(1 \rightarrow 4) \mathrm{bDGlcNAc}(1 \rightarrow 4)[\operatorname{aLFuc}(1 \rightarrow 6)] \mathrm{bDGlcNAc}(1 \rightarrow) \mathrm{N} 73$ \\
\hline N109C & FA2 & $\begin{array}{l}\operatorname{bDGlcNAc}(1 \rightarrow 2) \mathrm{aDMan}(1 \rightarrow 6)[\mathrm{bDGlcNAc}(1 \rightarrow 2) \operatorname{aDMan}(1 \rightarrow 3)] \mathrm{bDMan}(1 \rightarrow 4) \mathrm{bDGlcNAc}(1 \rightarrow 4)[\operatorname{aLFuc}(1 \rightarrow \\
\text { 6)]bDGlcNAc}(1 \rightarrow) \mathrm{N} 109\end{array}$ \\
\hline $\mathrm{N} 118 \mathrm{C}$ & FA2 & $\begin{array}{l}\operatorname{bDGlcNAc}(1 \rightarrow 2) \mathrm{aDMan}(1 \rightarrow 6)[\mathrm{bDGlcNAc}(1 \rightarrow 2) \mathrm{aDMan}(1 \rightarrow 3)] \mathrm{bDMan}(1 \rightarrow 4) \mathrm{bDGlcNAc}(1 \rightarrow 4)[\operatorname{aLFuc}(1 \rightarrow \\
\text { 6)]bDGlcNAc}(1 \rightarrow) \mathrm{N} 118\end{array}$ \\
\hline $\mathrm{N} 158 \mathrm{C}$ & M6 & $\begin{array}{l}\operatorname{aDMan}(1 \rightarrow 6)[\operatorname{aDMan}(1 \rightarrow 3)] \mathrm{aDMan}(1 \rightarrow 6)[\operatorname{aDMan}(1 \rightarrow 2) \mathrm{aDMan}(1 \rightarrow 3)] \mathrm{bDMan}(1 \rightarrow 4) \mathrm{bDGlcNAc}(1 \rightarrow \\
\text { 4)bDGlcNAc}(1 \rightarrow) \mathrm{N} 158\end{array}$ \\
\hline $\mathrm{N} 227 \mathrm{C}$ & M9 & $\begin{array}{l}\operatorname{aDMan}(1 \rightarrow 2) \mathrm{aDMan}(1 \rightarrow 6)[\operatorname{aDMan}(1 \rightarrow 2) \mathrm{aDMan}(1 \rightarrow 3)] \mathrm{aDMan}(1 \rightarrow 6)[\operatorname{aDMan}(1 \rightarrow 2) \mathrm{aDMan}(1 \rightarrow 2) \mathrm{aDMan}(1 \rightarrow \\
\text { 3)]bDMan }(1 \rightarrow 4) \mathrm{bDGlcNAc}(1 \rightarrow 4) \mathrm{bDGlcNAc}(1 \rightarrow) \mathrm{N} 227\end{array}$ \\
\hline $\mathrm{N} 269 \mathrm{C}$ & FA3G3S1 & $\begin{array}{l}\operatorname{aDNeu} 5 \operatorname{Ac}(2 \rightarrow 6) \mathrm{bDGal}(1 \rightarrow 4) \mathrm{bDGlcNAc}(1 \rightarrow 2) \mathrm{aDMan}(1 \rightarrow 3)[\mathrm{bDGal}(1 \rightarrow 4) \mathrm{bDGlcNAc}(1 \rightarrow 6)[\mathrm{bDGal}(1 \rightarrow \\
\text { 4)bDGlcNAc}(1 \rightarrow 2)] \mathrm{aDMan}(1 \rightarrow 6)] \mathrm{bDMan}(1 \rightarrow 4) \mathrm{bDGlcNAc}(1 \rightarrow 4)[\operatorname{aLFuc}(1 \rightarrow 6)] \mathrm{bDGlcNAc}(1 \rightarrow) \mathrm{N} 269\end{array}$ \\
\hline N318C & FA3G3S1 & $\begin{array}{l}\operatorname{aDNeu} 5 \operatorname{Ac}(2 \rightarrow 6) \mathrm{bDGal}(1 \rightarrow 4) \mathrm{bDGlcNAc}(1 \rightarrow 2) \mathrm{aDMan}(1 \rightarrow 3)[\mathrm{bDGal}(1 \rightarrow 4) \mathrm{bDGlcNAc}(1 \rightarrow 6)[\mathrm{bDGal}(1 \rightarrow \\
\text { 4)bDGlcNAc}(1 \rightarrow 2)] \mathrm{aDMan}(1 \rightarrow 6)] \mathrm{bDMan}(1 \rightarrow 4) \mathrm{bDGlcNAc}(1 \rightarrow 4)[\operatorname{aLFuc}(1 \rightarrow 6)] \mathrm{bDGlcNAc}(1 \rightarrow) \mathrm{N} 318\end{array}$ \\
\hline N330C & FA3G3S1 & $\begin{array}{l}\operatorname{aDNeu} 5 \operatorname{Ac}(2 \rightarrow 6) \mathrm{bDGal}(1 \rightarrow 4) \mathrm{bDGlcNAc}(1 \rightarrow 2) \mathrm{aDMan}(1 \rightarrow 3)[\mathrm{bDGal}(1 \rightarrow 4) \mathrm{bDGlcNAc}(1 \rightarrow 6)[\mathrm{bDGal}(1 \rightarrow \\
\text { 4)bDGlcNAc}(1 \rightarrow 2)] \mathrm{aDMan}(1 \rightarrow 6)] \mathrm{bDMan}(1 \rightarrow 4) \mathrm{bDGlcNAc}(1 \rightarrow 4)[\operatorname{aLFuc}(1 \rightarrow 6)] \mathrm{bDGlcNAc}(1 \rightarrow) \mathrm{N} 330\end{array}$ \\
\hline N357C & FA2 & $\begin{array}{l}\operatorname{bDGlcNAc}(1 \rightarrow 2) \mathrm{aDMan}(1 \rightarrow 6)[\mathrm{bDGlcNAc}(1 \rightarrow 2) \mathrm{aDMan}(1 \rightarrow 3)] \mathrm{bDMan}(1 \rightarrow 4) \mathrm{bDGlcNAc}(1 \rightarrow 4)[\operatorname{aLFuc}(1 \rightarrow \\
\text { 6)]bDGlcNAc}(1 \rightarrow) \mathrm{N} 357\end{array}$ \\
\hline N589C & M5 & $\begin{array}{l}\operatorname{aDMan}(1 \rightarrow 6)[\mathrm{aDMan}(1 \rightarrow 3)] \mathrm{aDMan}(1 \rightarrow 6)[\mathrm{aDMan}(1 \rightarrow 3)] \mathrm{bDMan}(1 \rightarrow 4) \mathrm{bDGlcNAc}(1 \rightarrow 4) \mathrm{bDGlcNAc}(1 \\
\rightarrow) \mathrm{N} 589\end{array}$ \\
\hline N602C & A3 & $\begin{array}{l}\operatorname{bDGlcNAc}(1 \rightarrow 6)[\mathrm{bDGlcNAc}(1 \rightarrow 2)] \mathrm{aDMan}(1 \rightarrow 6)[\mathrm{bDGlcNAc}(1 \rightarrow 2) \mathrm{aDMan}(1 \rightarrow 3)] \mathrm{bDMan}(1 \rightarrow \\
\text { 4)bDGlcNAc}(1 \rightarrow 4) \mathrm{bDGlcNAc}(1 \rightarrow) \mathrm{N} 602\end{array}$ \\
\hline N691C & FA2 & $\begin{array}{l}\operatorname{bDGlcNAc}(1 \rightarrow 2) \mathrm{aDMan}(1 \rightarrow 6)[\mathrm{bDGlcNAc}(1 \rightarrow 2) \mathrm{aDMan}(1 \rightarrow 3)] \mathrm{bDMan}(1 \rightarrow 4) \mathrm{bDGlcNAc}(1 \rightarrow 4)[\operatorname{aLFuc}(1 \rightarrow \\
\text { 6)]bDGlcNAc}(1 \rightarrow) \mathrm{N} 691\end{array}$ \\
\hline N699C & A3 & $\begin{array}{l}\operatorname{bDGlcNAc}(1 \rightarrow 6)[\operatorname{bDGlcNAc}(1 \rightarrow 2)] \mathrm{aDMan}(1 \rightarrow 6)[\operatorname{bDGlcNAc}(1 \rightarrow 2) \operatorname{aDMan}(1 \rightarrow 3)] \mathrm{bDMan}(1 \rightarrow \\
\text { 4)bDGlcNAc}(1 \rightarrow 4) \mathrm{bDGlcNAc}(1 \rightarrow) \mathrm{N} 699\end{array}$ \\
\hline N783C & M5 & $\begin{array}{l}\operatorname{aDMan}(1 \rightarrow 6)[\mathrm{aDMan}(1 \rightarrow 3)] \mathrm{aDMan}(1 \rightarrow 6)[\mathrm{aDMan}(1 \rightarrow 3)] \mathrm{bDMan}(1 \rightarrow 4) \mathrm{bDGlcNAc}(1 \rightarrow 4) \mathrm{bDGlcNAc}(1 \\
\rightarrow) \mathrm{N} 783\end{array}$ \\
\hline $\mathrm{N} 1056 \mathrm{C}$ & FA3G3S1 & $\begin{array}{l}\operatorname{aDNeu} 5 \operatorname{Ac}(2 \rightarrow 6) \mathrm{bDGal}(1 \rightarrow 4) \mathrm{bDGlcNAc}(1 \rightarrow 2) \mathrm{aDMan}(1 \rightarrow 3)[\mathrm{bDGal}(1 \rightarrow 4) \mathrm{bDGlcNAc}(1 \rightarrow 6)[\mathrm{bDGal}(1 \rightarrow \\
\text { 4)bDGlcNAc}(1 \rightarrow 2)] \mathrm{aDMan}(1 \rightarrow 6)] \mathrm{bDMan}(1 \rightarrow 4) \mathrm{bDGlcNAc}(1 \rightarrow 4)[\operatorname{aLFuc}(1 \rightarrow 6)] \mathrm{bDGlcNAc}(1 \rightarrow) \mathrm{N} 1056\end{array}$ \\
\hline N1080C & A2 & $\begin{array}{l}\text { bDGlcNAc( }(1 \rightarrow 2) \mathrm{aDMan}(1 \rightarrow 6)[\mathrm{bDGlcNAc}(1 \rightarrow 2) \mathrm{aDMan}(1 \rightarrow 3)] \mathrm{bDMan}(1 \rightarrow 4) \mathrm{bDGlcNAc}(1 \rightarrow 4) \mathrm{bDGlcNAc}(1 \\
\rightarrow) \mathrm{N} 1080\end{array}$ \\
\hline
\end{tabular}

Table S7. Components of the binding free energy ( $\mathrm{kcal} / \mathrm{mol})$ calculated for glycosylated CoV-2-S with small and large RBD-angles*

\begin{tabular}{lcc}
\hline Energy term & $6 \mathrm{ACG}\left(54.5^{\circ} \pm 0.86\right)$ & $6 \mathrm{ACK}\left(84.6^{\circ} \pm 0.77\right)$ \\
\hline$E_{V D W}$ & $-92.22 \pm 0.86$ & $-100.78 \pm 0.91$ \\
$E_{\text {ele }}$ & $-915.87 \pm 2.95$ & $-767.25 \pm 3.93$ \\
$E_{g b}$ & $983.72 \pm 2.90$ & $824.33 \pm 3.82$ \\
$E_{n p}$ & $-13.07 \pm 0.08$ & $-14.60 \pm 0.10$ \\
$\Delta H$ & $-37.46 \pm 0.70$ & $-58.31 \pm 0.74$
\end{tabular}




$\begin{array}{lll}-T \Delta S & -18.32 \pm 0.74 & -20.58 \pm 0.96 \\ \Delta G & -19.14 \pm 0.72 & -37.73 \pm 0.85\end{array}$

*: The statistical error was estimated based on 50-100 ns MD simulation trajectory. A total of 1000 snapshots evenly extracted from the 50-100 ns MD trajectory of each complex were used for MM/GBSA calculations, and 10 snapshots for the entropy term calculations.

Table S8. The predicted ACE2-binding free energy ( $\mathrm{kcal} / \mathrm{mol})$ for $\mathrm{CoV}-\mathrm{S}$ and $\mathrm{CoV}-2-$ $\mathrm{S}$ trimers based on three different $\varepsilon_{\mathrm{in} .}{ }^{\mathrm{a}}$

\begin{tabular}{lllllll}
\hline Organism & \multicolumn{2}{c}{ CoV-S } & \multicolumn{3}{c}{ CoV-2-S } \\
\hline$\varepsilon_{\text {in }}$ & 1 & 2 & 4 & 1 & 2 & 4 \\
6 ACG & $-10.59 \pm 0.62$ & $-22.70 \pm 0.88$ & $-31.49 \pm 0.97$ & $-18.00 \pm 0.84$ & $-24.47 \pm 0.86$ & $-33.95 \pm 0.99$ \\
6 ACJ & $-12.39 \pm 0.68$ & $-23.65 \pm 0.95$ & $-30.28 \pm 0.82$ & $-25.10 \pm 0.68$ & $-35.21 \pm 0.82$ & $-43.22 \pm 1.13$ \\
6 CS2 & $-11.54 \pm 0.72$ & $-22.37 \pm 1.01$ & $-32.04 \pm 0.90$ & $-32.40 \pm 0.86$ & $-39.57 \pm 0.58$ & $-45.10 \pm 0.75$ \\
6 ACK & $-20.63 \pm 0.60$ & $-37.15 \pm 0.95$ & $-45.48 \pm 0.97$ & $-39.52 \pm 0.61$ & $-54.01 \pm 1.02$ & $-57.77 \pm 1.43$ \\
\hline
\end{tabular}

a: The statistical error was estimated based on 50-100 ns MD simulation trajectory. 1000 snapshots evenly extracted from the 50-100 ns MD trajectory of each complex were used for MM/GBSA calculations. 


\section{References}

1. Li, F.; Li, W.; Michael, F.; Harrison, S. C. Structure of SARS Coronavirus Spike ReceptorBinding Domain Complexed with Receptor. Science 2005, 309 (5742), 1864-1868.

2. Song, W.; Gui, M.; Wang, X.; Xiang, Y. Cryo-EM structure of the SARS coronavirus spike glycoprotein in complex with its host cell receptor ACE2. PLoS pathogens 2018, 14 (8), e1007236.

3. Kirchdoerfer, R. N.; Wang, N.; Pallesen, J.; Wrapp, D.; Turner, H. L.; Cottrell, C. A.; Corbett, K. S.; Graham, B. S.; McLellan, J. S.; Ward, A. B. Stabilized coronavirus spikes are resistant to conformational changes induced by receptor recognition or proteolysis. Sci Rep 2018, $8(1), 15701$.

4. Lan, J.; Ge, J.; Yu, J.; Shan, S.; Zhou, H.; Fan, S.; Zhang, Q.; Shi, X.; Wang, Q.; Zhang, L. et al. Structure of the SARS-CoV-2 spike receptor-binding domain bound to the ACE2 receptor. Nature 2020, 581 (7807), 215-220.

5. Waterhouse, A.; Bertoni, M.; Bienert, S.; Studer, G.; Tauriello, G.; Gumienny, R.; Heer, F. T.; de Beer, T. A. P.; Rempfer, C.; Bordoli, L. et al. SWISS-MODEL: homology modelling of protein structures and complexes. Nucleic Acids Res 2018, 46 (W1), W296-W303.

6. Duan, Y.; Wu, C.; Chowdhury, S.; Lee, M. C.; Xiong, G.; Zhang, W.; Yang, R.; Cieplak, P.; Luo, R.; Lee, T. et al. A Point-charge Force Field for Molecular Mechanics Simulations of Proteins Based on Condensed-Phase Quantum Mechanical Calculations J Comput Chem 2003, 24, 1999-2012.

7. Ryckaert, J. P.; Ciccotti, G.; Berendsen, H. J. C. Numerical integration of the cartesian equations of motion of a system with constraints: molecular dynamics of n-alkanes. J Comput Phys 1977, 23 (3), 327-341.

8. Essmann, U. P., L.; Berkowitz, M. L.; Darden, T.; Lee, H.; Pedersen, L. G. A smooth particle mesh Ewald method. J Comput Phys 1995, 103, 8577-8593.

9. Wang, J.; Peng, C.; Yu, Y.; Chen, Z.; Xu, Z.; Cai, T.; Shao, Q.; Shi, J.; Zhu, W. Exploring Conformational Change of Adenylate Kinase by Replica Exchange Molecular Dynamic Simulation. Biophys J 2020, 118 (5), 1009-1018.

10. Onufriev, A.; Bashford, D.; Case, D. A. Exploring protein native states and large-scale conformational changes with a modified generalized born model. Proteins 2004, 55 (2), 383-394. 11. Chen, F.; Liu, H.; Sun, H.; Pan, P.; Li, Y.; Li, D.; Hou, T. Assessing the performance of the MM/PBSA and MM/GBSA methods. 6. Capability to predict protein-protein binding free energies and re-rank binding poses generated by protein-protein docking. Phys Phem Phem Phys 2016, 18 (32), 22129-39.

12. Wang, J.; Shao, Q.; Xu, Z.; Liu, Y.; Yang, Z.; Cossins, B. P.; Jiang, H.; Chen, K.; Shi, J.; $\mathrm{Zhu}, \mathrm{W}$. Exploring transition pathway and free-energy profile of large-scale protein conformational change by combining normal mode analysis and umbrella sampling molecular dynamics. J Phys Chem B 2014, 118 (1), 134-43.

13. Yuan, Y.; Cao, D.; Zhang, Y.; Ma, J.; Qi, J.; Wang, Q.; Lu, G.; Wu, Y.; Yan, J.; Shi, Y. et al. Cryo-EM structures of MERS-CoV and SARS-CoV spike glycoproteins reveal the dynamic receptor binding domains. Nat Commun 2017, 8, 15092.

14. Gungor, O.; Edward, F. V.; Stephen, Q.; Hernandez, R. Adaptive Steered Molecular Dynamics of the Long-Distance Unfolding of Neuropeptide Y. J Chem Theory Comput 2010, 6, 
3026-3038.

15. Herrera, N. G.; Morano, N. C.; Celikgil, A.; Georgiev, G. I.; Malonis, R. J.; Lee, J. H.; Tong, K.; Vergnolle, O.; Massimi, A. B.; Yen, L. Y. et al. Characterization of the SARS-CoV-2 S Protein: Biophysical, Biochemical, Structural, and Antigenic Analysis. bioRxiv 2020.

16. Wrapp, D.; Wang, N.; Corbett, K. S.; Goldsmith, J. A.; Hsieh, C.; Abiona, O.; Graham, B. S.; McLellan, J. S. Cryo-EM structure of the 2019-nCoV spike in the prefusion conformation. Science 2020, 367, 1260-1263.

17. Walls, A. C.; Park, Y. J.; Tortorici, M. A.; Wall, A.; McGuire, A. T.; Veesler, D. Structure, Function, and Antigenicity of the SARS-CoV-2 Spike Glycoprotein. Cell 2020, 181 (2), 281-292 e6.

18. Pinto, D.; Park, Y. J.; Beltramello, M.; Walls, A. C.; Tortorici, M. A.; Bianchi, S.; Jaconi, S.; Culap, K.; Zatta, F.; De Marco, A. et al. Structural and functional analysis of a potent sarbecovirus neutralizing antibody. bioRxiv 2020.

19. Henderson, R.; Edwards, R. J.; Mansouri, K.; Janowska, K.; Stalls, V.; Gobeil, S.; Kopp, M.; Hsu, A.; Borgnia, M.; Parks, R. et al. Controlling the SARS-CoV-2 Spike Glycoprotein Conformation. bioRxiv 2020.

20. Barnes, C. O.; West A. P., Jr.; Huey-Tubman, K. E.; Hoffmann, M. A. G.; Sharaf, N. G.; Hoffman, P. R.; Koranda, N.; Gristick, H. B.; Gaebler, C.; Muecksch, F. et al. Structures of Human Antibodies Bound to SARS-CoV-2 Spike Reveal Common Epitopes and Recurrent Features of Antibodies. Cell 2020, 182 (4), 828-842.

21. Liu, L.; Wang, P.; Nair, M. S.; Yu, J.; Rapp, M.; Wang, Q.; Luo, Y.; Chan, J. F.; Sahi, V.; Figueroa, A. et al. Potent Neutralizing Monoclonal Antibodies Directed to Multiple Epitopes on the SARS-CoV-2 Spike. bioRxiv 2020.

22. Hsieh, C. L.; Goldsmith, J. A.; Schaub, J. M.; DiVenere, A. M.; Kuo, H. C.; Javanmardi, K.; Le, K. C.; Wrapp, D.; Lee, A. G.; Liu, Y. et al. Structure-based Design of Prefusion-stabilized SARS-CoV-2 Spikes. bioRxiv 2020.

23. Zhou, T.; Tsybovsky, Y.; Olia, A. S.; Gorman, J.; Rapp, M. A.; Cerutti, G.; Katsamba, P. S.; Nazzari, A.; Schon, A.; Wang, P. D. et al. A pH-dependent switch mediates conformational masking of SARS-CoV-2 spike. bioRxiv 2020.

24. Cai, Y. F.; Zhang, J.; Xiao, T. S.; Peng, H. Q.; Sterling, S. M.; Walsh Jr, R. M.; Rawson, S.; Rits-Volloch, S.; Chen, B. Distinct conformational states of SARS-CoV-2 spike protein. Science 2020, 369 (6511), 1586-1592.

25. Yurkovetskiy, L.; Pascal, K. E.; Tompkins-Tinch, C.; Nyalile, T.; Wang, Y.; Baum, A.; Diehl, W. E.; Dauphin, A.; Carbone, C.; Veinotte, K. et al. SARS-CoV-2 Spike protein variant D614G increases infectivity and retains sensitivity to antibodies that target the receptor binding domain. bioRxiv 2020.

26. Zhe, L.; Deng, Y. Q.; Ye, Q.; Cao, L.; Sun, C. Y.; Fan, C.; Huang, W.; Sun, S.; Sun, Y.; Zhu, L. et al. Structural basis for neutralization of SARS-CoV-2 and SARS-CoV by a potent therapeutic antibody. Science 2020, 369 (6510), 1505-1509.

27. Huo, J.; Zhao, Y.; Ren, J.; Zhou, D.; Duyvesteyn, H. M. E.; Ginn, H. M.; Carrique, L.; Malinauskas, T.; Ruza, R. R.; Shah, P. N. M. et al. Neutralization of SARS-CoV-2 by Destruction of the Prefusion Spike. Cell Host Microbe 2020.

28. Wrobel, A. G.; Benton, D. J.; Xu, P.; Roustan, C.; Martin, S. R.; Rosenthal, P. B.; Skehel, J. J.; Gamblin, S. J. Evolution of SARS-CoV-2 spike glycoprotein. Research Square 2020. 
29. Melero, R.; Sorzano, C. O. S.; Foster, B.; Vilas, J. L.; Martinez, M.; Marabini, R.; RamirezAportela, E.; Sanchez-Garcia, R.; Herreros, D.; Del Cano, L. et al. Continuous flexibility analysis of SARS-CoV-2 Spike prefusion structures. bioRxiv 2020.

30. Xiong, X.; Qu, K.; Ciazynska, K. A.; Hosmillo, M.; Carter, A. P.; Ebrahimi, S.; Ke, Z.;

Scheres, S. H. W.; Bergamaschi, L.; Grice, G. L. et al. A thermostable, closed SARS-CoV-2 spike protein trimer. Nat Struct Mol Biol 2020.

31. Cao, Y.; Su, B.; Guo, X.; Sun, W.; Deng, Y.; Bao, L.; Zhu, Q.; Zhang, X.; Zheng, Y.; Geng, C. et al. Potent Neutralizing Antibodies against SARS-CoV-2 Identified by High-Throughput Single-Cell Sequencing of Convalescent Patients' B Cells. Cell 2020, 182 (1), $73-84$ e16.

32. Chi, X; Yan, R; Zhang, J. Zhang, G. Zhang, Y. Hao, M.; Zhang, Z. Fan, P.; Dong, Y.; Yang, Y. et al. A neutralizing human antibody binds to the N-terminal domain of the Spike protein of SARS-CoV-2. Science 2020, 369 (6504), 650-655.

33. Gui, M.; Song, W.; Zhou, H.; Xu, J.; Chen, S.; Xiang, Y.; Wang, X. Cryo-electron microscopy structures of the SARS-CoV spike glycoprotein reveal a prerequisite conformational state for receptor binding. Cell Res 2017, 27 (1), 119-129.

34. Walls, A. C.; Xiong, X.; Park, Y. J.; Tortorici, M. A.; Snijder, J.; Quispe, J.; Cameroni, E.; Gopal, R.; Dai, M.; Lanzavecchia, A. et al. Unexpected Receptor Functional Mimicry Elucidates Activation of Coronavirus Fusion. Cell 2019, 176 (5), 1026-1039 e15.

35. Kao, R. Y.; Tsui, W. H.; Lee, T. S.; Tanner, J. A.; Watt, R. M.; Huang, J. D.; Hu, L.; Chen, G.; Chen, Z.; Zhang, L. et al. Identification of novel small-molecule inhibitors of severe acute respiratory syndrome-associated coronavirus by chemical genetics. Chem Biol 2004, 11 (9), 12931299.

36. Yi, L.; Li, Z.; Yuan, K.; Qu, X.; Chen, J.; Wang, G.; Zhang, H.; Luo, H.; Zhu, L.; Jiang, P. et al. Small molecules blocking the entry of severe acute respiratory syndrome coronavirus into host cells. J Virol 2004, 78 (20), 11334-11339.

37. Wang, X.; Cao, R.; Zhang, H.; Liu, J.; Xu, M.; Hu, H.; Li, Y.; Zhao, L.; Li, W.; Sun, X. et al. The anti-influenza virus drug, arbidol is an efficient inhibitor of SARS-CoV-2 in vitro. Cell Discov 2020, 6, 28.

38. Zhao, G.; Du, L.; Ma, C.; Li, Y.; Li, L.; Poon, V. K.; Wang, L.; Yu, F.; Zheng, B. J.; Jiang, S. et al. A safe and convenient pseudovirus-based inhibition assay to detect neutralizing antibodies and screen for viral entry inhibitors against the novel human coronavirus MERS-CoV. Virol J 2013, 10, 266.

39. Kim, J. Y.; Kim, Y. I.; Park, S. J.; Kim, I. K.; Choi, Y. K.; Kim, S. H. Safe, high-throughput screening of natural compounds of MERS-CoV entry inhibitors using a pseudovirus expressing MERS-CoV spike protein. Int $J$ Antimicrob Agents 2018, 52 (5), 730-732.

40. Adedeji, A. O.; Severson, W.; Jonsson, C.; Singh, K.; Weiss, S. R.; Sarafianos, S. G. Novel inhibitors of severe acute respiratory syndrome coronavirus entry that act by three distinct mechanisms. J Virol 2013, 87 (14), 8017-8028.

41. Casalino, L.; Gaieb, Z.; Goldsmith, J. A.; Hjorth, C. K.; Dommer, A. C.; Harbison, A. M.; Fogarty, C. A.; Barros, E. P.; Taylor, B. C.; McLellan, J. S. et al. Beyond Shielding: The Roles of Glycans in the SARS-CoV-2 Spike Protein. ACS Cent Sci 2020, 6 (10), 1722-1734.

42. Yasunori, W.; Joel, D. A.; Daniel, W.; Jason, S. M.; Crispin, M. Site-specific glycan analysis of the SARS-CoV-2 spike. Science 2020, 369 (6501), 330-333.

43. Watanabe, Y.; Berndsen, Z. T.; Raghwani, J.; Seabright, G. E.; Allen, J. D.; Pybus, O. G.; 
McLellan, J. S.; Wilson, I. A.; Bowden, T. A.; Ward, A. B. et al. Vulnerabilities in coronavirus glycan shields despite extensive glycosylation. Nat Commun 2020, 11 (1), 2688. 